\title{
Potential of Prospective Medicinal Plants of Bangladesh for the Complementary Management of COVID-19
}

\author{
Saimon Shahriar ${ }^{1}$, Md. Moklesur Rahman Sarker², Mahfuza Afroz Soma ${ }^{2}$, \\ Tabassum Jannat ${ }^{2}$, Sadia Tasnim Mina ${ }^{3}$, Rabita Rahman ${ }^{3}$, \\ Mohammad Sharifur Rahman ${ }^{1}$ and Mohammad A. Rashid ${ }^{1 *}$
}

\author{
${ }^{1}$ Department of Pharmaceutical Chemistry, Faculty of Pharmacy, University of Dhaka, Dhaka 1000, Bangladesh \\ ${ }^{2}$ Department of Pharmacy, State University Bangladesh, 77 Satmasjid Road, Dhanmondi, \\ Dhaka-1205, Bangladesh \\ ${ }^{3}$ Department of Pharmacy, Faculty of Pharmacy, University of Dhaka, Dhaka 1000, Bangladesh
}

(Received: September 9, 2021; Accepted: December 15, 2021; Published (web): January 29, 2022)

\begin{abstract}
:
The COVID-19 pandemic, caused by the SARS-CoV-2 virus, has been a healthcare disaster because of the unique and distinct characteristics of the pathogen, the easy and rapid transmission of the virus from humans to humans, the challenges in diagnosis and confirmation of the disease and the inability to invent and distribute safe and effective drugs or vaccines worldwide that would work against all the variants of coronavirus. Bangladesh, despite being a third-world country with limited health resources, has not been one of the worst-hit countries in the world but has still suffered with the loss of nearly eleven thousand people. Traditional and herbal remedies have become popular in this sub-continent since long ago and used for the treatment and management of different diseases including infectious disease. In this review, we have summarized the reports of immunostimulating, anti-inflammatory, antiviral, and respiratory distress syndrome improving activities of prospective indigenous plants of Bangladesh that may be recommended for use as complementary and alternative medicine or may be potential sources for the discovery and development of anti-COVID-19 medicaments. Thus, the review will be beneficial for the researchers, complementary and alternative medicines or herbal medicine manufacturers, formulators to find out and manage the potential herbal/nutraceutical/medicinal agents for the preparation of complementary and alternative medicines, as well as to the scientist for further research for the discovery and development of therapeutics/new drugs for the prevention and treatment of COVID-19 as well as other viral infections.
\end{abstract}

Keywords: COVID-19, SARS-CoV-2, traditional medicine, herbal medicine, immunomodulation, antiviral, anti-inflammatory, complementary and alternative medicine, medicinal plants, nutraceuticals, bioactive phytocompounds, Bangladesh

\section{Introduction}

A unique virus emerged in China at the end of 2019 and spread the COVID-19 (coronavirus disease2019) outside China by February 2020 and became a pandemic ravaging the whole world (Israfil et al., 2021; Shahriar and Koly, 2021). The virus was named severe acute respiratory syndrome coronavirus-2 (SARS-CoV-2) and was different from other coronaviruses, which caused the SARS (severe acute respiratory syndrome) and MERS (middle eastern respiratory syndrome) outbreak. Bangladesh has been hit hard by this deadly disease too (Hossain et al., 2020; Hossain and Rahman, 2020). This disease has claimed the lives of approximately eleven thousand people in Bangladesh and over three million people worldwide (Worldometer, 2021).

*Corresponding authors: Md. Moklesur Rahman Sarker, Department of Pharmacy, State University of Bangladesh, 77 Satmasjid Road, Dhanmondi, Dhaka 1205, Bangladesh

Email:moklesur2002@yahoo.com; prof.moklesur@sub.edu.bd

Mohammad A. Rashid, Department of Pharmaceutical Chemistry, Faculty of Pharmacy, University of Dhaka, Dhaka 1000, Bangladesh. Email: r.pchem@yahoo.com 
SARS-CoV-2 is a member of the family Coronaviridae and the order Nidovirales. It has a spike protein resembling the shape of a crown, and hence, it has the name coronavirus ('corona' means crown). It has an incubation period of 5-6 days, extending up to 14 days in some cases. It is spread through the droplets of an infected person who may or may not be symptomatic. The droplets can be transmitted through handshake and by being in close contact with other infected persons (Polak et al., 2020; Shahriar et al., 2021).

The symptoms of COVID-19 include dry cough, fever, malaise, and headache in mild cases. However, complicated cases are presented with breathlessness, low blood oxygen level, which may lead to hypoxia, the collapse of the respiratory system, and multiorgan failure in severe cases. Its mild symptoms often coincide with those of common cold and flu, but can be confirmed through reverse transcriptase polymerase chain reaction (RT-PCR) testing or a chest $\mathrm{CT}$ (computer tomography). The virus causes direct cell injury as well as indirect cellular damage through immune system dysfunction. Its virulence depends on the non-structural proteins (NSPS) and structural proteins including the spike glycoprotein (S). Besides, the main protease, papain-like protease, helicase, and RNA-dependent RNA polymerase enzymes also participate in the pathophysiological mechanism (Hu et al., 2020; Shahriar et al., 2021).

At the beginning of COVID-19 pandemic, antimicrobial and prospective other drugs were used as repurposed-drugs for the treatment of COVID-19 but those drugs were found to have limited efficacy and several adverse-effects (Alam et al., 2021a). There has been no drug invented that can be used safely and effectively to treat COVID-19 in all patients. The newly developed vaccines have shown some adverse-effects (Hossain et al., 2021; Hossain and Rahman, 2020; Alam et al., 2021b; Sultana et al., 2021) and are not found to be $100 \%$ effective in controlling the spread of COVID-19 infection. In these circumstances, traditional and herbal medicinal plants can be of great interest. Ayurveda, the ancient Indian medicine system, showed us that it could lead modern scientists towards better medicines from natural sources (Alam et al., 2021b). The traditional Chinese medicine (TCM) system can be of similar help as well (Fabricant and Farnsworth, 2001; Graziose et al., 2010; Khanna et al., 2020). Phytomedicines, medicinal plants, and functional foods are valuable sources for the discovery of lead compounds as well as using as herbal formulations, i.e. antidiabetic, anticancer, anti-inflammatory, immunomodulating, anti-HIV, antioxidant, etc. (Alam et al., 2020; Kaisar et al., 2011; Rahman et al., 2011; Rashid et al., 2000; Rouhi et al., 2017; Sarker and Gohda, 2013; Sarker, 2012; Sarker et al., 2011; Sarker et al., 2015; Sarker et al., 2016). Being a tropical country, Bangladesh is a rich source of medicinal plants. COVID-19 infection is prominently marked by acute respiratory distress syndrome, severe inflammation, vascular edema, multiple organ failure, etc. Immuno-compromised people suffer a lot (Shahriar et al., 2021). Exploring the immunomodulating and antiviral activities, indigenous plants of Bangladesh can be a great source of remedies, especially for the prevention and treatment of COVID-19 infections to an extent (Sarker, 2021). In this review, we have discussed the scientific basis and potentiality of notable medicinal plants of Bangladesh that can be used for the prevention and treatment of COVID-19 infections.

\section{Article Search Strategy}

To collect data on medicinal plants of Bangladesh having therapeutic value for the prevention and treatment of COVID-19 and/or COVID-19 related complications, a literature search was performed throughout the PubMed, Web of Science, Scopus, ScienceDirect, Wiley Online Library, and Google Scholar databases. The following keywords were used while searching articles on the above databases: "COVID-19", "SARS-CoV-2", "phytomedicines", "bioactive compounds", "traditional medicinal plants", "Bangladesh", "indigenous medicinal plants", "herbal medicine," "anti-COVID-19", "antiinflammatory", "anti-coagulating", "antiviral" "immunostimulating" and "immunomodulating". 
Articles published in English language only were considered.

\section{Prospective indigenous plants of Bangladesh with immunoboosting and antiviral activities}

In this review, we have briefly presented some prospective medicinal plants of Bangladesh that were previously reported to exhibit anti-inflammatory, antiviral, immunostimulanting and respiratory distress syndrome improving properties. All the possible prospective plants have been summarized Table 1 along with their activity reports. It has shown us that the different parts of the plant, extracts, and isolated compounds have the potentials against inflammation, respiratory distress syndrome, viral infections, etc. Some in-silico studies have also shown promising ground to work against the coronavirus. The structures of some major bioactive compounds from these plants are also presented in Figure 1.

Basil (Ocimum sanctum L., Family: Lamiaceae): Basil ('Tulsi' in Bengali language) is a holy herb according to the Hindu religion. Its medicinal property is well-reported. It has immunomodulatory, anti-inflammatory, antiviral, and adaptogenic effects. 'Tulsi' leaves are often called as 'elixir of life'. Several 'Tulsi' constituents including vicenin, ursolic acid, tulsinol A-G and isorientin 4'-O-glucoside 2"$O$-p-hydroxybenzoagte showed strong inhibiting property against SARS-CoV-2 virus in-silico (Shree et al., 2020; Varshney et al., 2020). Using 'Tulsi' leaf preparations may be a beneficial choice against COVID-19.

Black cumin (Nigella sativa L., Family: Ranunculaceae): Black cumin or black caraway ('Kalojira' in Bengali language) is a frequently used spice and a medicinal plant. Nigella sativa has been reported to show the immunomodulatory, antiviral, and anti-inflammatory activities (Sarker et al., 2011). It has a number of bioactive constituents of different types including alkaloids, saponins, and essential oils. Several in-silico studies reported that its constituents, e.g., nigelledine, hederagenin, and $\alpha$-hederin might be used against SARS-CoV-2 (Koshak and Koshak, 2020). Zinc supplement efficiency in managing
COVID-19 might be enhanced by black cumin (Rahman, 2020). The antiviral activity of this plant makes it a likely candidate for herbal medicine against COVID-19.

Black mulberry (Morus nigra L., Family: Moraceae): Black mulberry ('Toot' in Bengali language) contains phenolic glycosides, prenylated flavonoids, terpenoids, coumarins etc. The jam made of berry fruits has been traditionally used to treat inflammatory diseases, like- cough, asthma, other chest complaints and rheumatism (Lim and Choi, 2019; Nomura, 1988). During COVID-19 pandemic, unani physicians have prescribed gargling with a solution of toot before sleep in the treatment of respiratory distresses (Nikhat and Fazil, 2020).

Black Pepper (Piper nigrum L., Family: Piperaceae): Black pepper ('Gol Morich' in Bengali language) fruit is a popular spice. Studies reported that it displayed analgesic, antipyretic and CNS stimulant activities (Emon et al., 2021). A study reported that it might be used as an immune booster in COVID-19. The anti-SARS-CoV-2 activity was assumed to take place through piperine, a major constituent of black pepper. It showed higher binding potential with the virus nucleocapsid and subsequent decrease of the virulence (Choudhary et al., 2020).

Caraway (Trachyspermum ammi L. Sprague, Family: Apiaceae): Caraway seeds, also known as Ajwain ('Mouri' in Bengali language), has been proven to be effective in sore throat/dry cough and eradicating infection (Banerjee et al., 2020). Its oil has shown antiviral activity against encephalitis virus in-vitro (Roy et al., 2015). Its immune-boosting activity is also well-reported (Vitali et al., 2016). It can be proposed that its nutritional benefit may extend to the case of COVID-19.

Chebulic Myrobalan (Terminalia chebula Retz., Family: Combretaceae): Chebulic Myrobalan ('Haritaki' in Bengali language) is a widely-known medicinal plant. Previous studies reported that it showed antiviral, antioxidant and anti-inflammatory potential. Several compounds isolated from this plant showed strong activity against a number of viruses including human immunodeficiency virus (HIV), 
hepatitis $\mathrm{B}$ and $\mathrm{C}$ virus, and influenza A virus (Ajala et al., 2014; Ma et al., 2010). It is likely that this plant may boost the immunity of the people and help them in this pandemic (Haque et al., 2021).

Citron (Citrus medica L., Family: Rutaceae): Citron ('Jamir Lebu' in Bengali language) is a common fruit in Bangladesh. Its peels were reported to have phenolic compounds, ascorbic acid, iron, zinc, and other minerals. These constituents have showed immune-boosting activity in several studies (Haridas et al., 2021; Karetha et al., 2020). Hence, citron may also be used as an immune-booster in this pandemic.

Clove (Syzygium aromaticum L. Merr., Family: Myrtaceae): Clove ('Labongo' in Bengali language) is also a common spice with reports of anthelmintic, antimicrobial and other activities. It was administered alone and with other herbs like metha and citrus to COVID-19 infected patients and results showed that those preparations had immune-boosting properties. Therefore, this plant may be used as a herbal immune booster alone or with other herbs and may help against COVID-19 related complications (Kanyinda, 2020).

Coconut (Cocos nucifera L., Family: Arecaceae): Coconut ('Narkel' in Bengali language) is a popular fruit in Bangladesh and its juice is frequently used as a drink. It may also be used as a vegetable. Previous studies reported that fruit and fiber extracts showed antioxidant, antiviral, and antibacterial activities. It was thought to be used in COVID-19 as well. A compound from coconut fruit, $\alpha$-tocotrienol, was found to have the potential to inhibit COVID-19 progression in-silico (Fitriani et al., 2020). Besides, in-vitro antiviral activity against herpes simplex virus was also displayed by the fiber extracts of coconut (Esquenazi et al., 2002). Its use in everyday life may have rewarding consequences.

Creat (Andrographis paniculata (Burm.f.) Nees., Family: Acanthaceae): Creat ('Kalomegh' or 'Chirotta' in Bengali language) is a widely used medicinal plant. It has anti-inflammatory, antihypertensive, anti-cancer and hepatoprotective properties. Dihydroxy dimethoxy flavone, andrographolide, and neoandrographolide, isolated from this plant, showed strong binding affinity for the SARS-CoV-2 virus active site in-silico (Murugan et al., 2020; Rajagopal et al., 2020; Sukardiman et al., 2020). Creat extract also showed immune-boosting and antiviral activity in-vitro against retro virus (Churiyah et al., 2015). Using 'Kalomegh' preparations for protecting one's heath against COVID-19 may be beneficial.

Flaxseed (Linum usitatissimum L., Family: Linaceae): Flaxseed ('Tishi' in Bengali language) has been proven to be a magical food responsible for several potential actions like antihypertensive, cholesterol lowering, antiatherogenic, antiinflammatory, and relief of arrythmia (Parikh et al., 2018). The main constituent responsible for the variety of activities is proven to be $\alpha$-linoleic acid, also known as omega-3 fatty acid, which is extremely beneficial for the treatment of arterial disorders and asthma. Thus, flaxseed may possess some activity against SARS-CoV-2 infection by acting as an immune booster (Samal et al., 2020).

Garlic (Allium sativum L., Family: Amaryllidaceae): Garlic (Roshun in Bengali language) is a common condiment. Studies reported that it as well as its constituents, e.g., allicin and ajoene, displayed antiviral property against influenza $A$ and $B$ virus, HIV, rhinovirus and herpes simplex virus. Computational study found that a number of its constituents showed potential activity against SARSCoV-2. Its anti-COVID-19 activity may work through constituents like quercetin, allicin and alliin (organosulfur and flavonoid compounds) (Khubber et al., 2020; Pandey et al., 2021). It also displayed immune-boosting activity which functioned through the enhancement of the activity and number of natural killer cells and several cytokines (Wang et al., 2010). Daily intake of garlic may boost the immune system and protect from this disease (Donma and Donma, 2020).

Giloy (Tinospora cordifolia (Willd.) Miers, Family: Menispermaceae): Giloy ('Guloncho' in Bengali language) is popular medicinal plant which has been used to treat disease like rheumatism, 
inflammation, allergy and jaundice. Studies also reported that it had immune-booster effects. Berberine and tinocordiside, major constituents of 'Giloy', showed potential for inhibition of viral replication of SARS-CoV-2 in silico through modulating viral protease activity (Chowdhury, 2020; Kumar and Chander, 2020; Shree et al., 2020).

Ginger (Zingiber officinale L., Family: Zingiberaceae): Ginger ('Ada' in Bangali language) is a 'generally recognized as safe' (GRAS) spice which has been reported to possess antiinflammatory, antioxidant, anti-emetic, bronchoprotective, and several other properties (Mao et al., 2019). A study reported moderate reduction of inflammatory mediators like tumor necrosis factor- $\alpha$ (TNF- $\alpha$ ) and interleukin-6 (IL-6) by ginger preparations resulting in a reduction of exhaustion, inflammation and infection (Magzoub, 2020). Besides, humoral immunity was also found to be boosted by ginger extracts (Carrasco et al., 2009). It is likely that ginger may boost the immune system against SARS-CoV-2 through its anti-inflammatory, antioxidant and immunomodulatory activities.

Golden shower tree (Cassia fistula L., Family: Fabaceae): Golden shower tree ('Shonalu' in Bangali language) is a flowering plant found in tropical and sub-tropical parts of the world. The plant possesses potential immunomodulatory, antioxidant, hepatoprotective, anti-inflammatory properties etc. (Chaerunisaa et al., 2020; Rahmani, 2015). Moreover, standardized formulations of this plant have been used as hand sanitizers and soaps (Killedar and Nale, 2014). A study reported significant stimulatory effect of $C$. fistula in cell-mediated immunity on rats without affecting the humoral immunity (Jadhav, 2014). In 2020, Tiwari Pandey et al. postulated that its leaf extract possesses antiinfective potencies against multidrug resistant strains reported in co-infections with bacterial and fungal origin (Tiwari et al., 2020). Therefore, C. fistula would be an effective treatment option in COVID-19 associated co-infections.

Gymnema (Gymnema sylvestre R. Br., Family: Apocyanaceae): Gymnema ('Gurmar' in Bengali language) has been used as a medicinal plant in Bangladesh. It has been known for its antiinflammatory, immunomodulatory, and antioxidant activity (Saneja et al., 2010). Its antiviral activity is also well-reported (Porchezhian and Dobriyal, 2003). Phytochemicals of this plant have the potential to be used in COVID-19 treatment, as found in a computational study (Subramani et al., 2020). Its anti-COVID-19 activity may be characterized through its antioxidant and immunomodulatory properties.

Indian Ginseng (Withania somnifera L. Dunal, Family: Solanaceae): Indian Ginseng, which is also called Poison Gooseberry, ('Ashwagandha' in Bengali language) is a common medicinal plant in the Indian subcontinent and known as a rejuvenator and longevity enhancer in Ayurveda, ancient Indian medicine system. This plant was reported to possess anti-inflammatory, neuroprotective, immunomodulatory, and anti-cancer properties. In silico studies reported that somniferine and withanoside $\mathrm{V}$, compounds isolated from this plant, showed strong potential for inhibiting COVID-19 disease progression (Shree et al., 2020; Srivastava et al., 2020). Its extracts showed effectiveness against herpes simplex virus (Kambizi et al., 2007). This plant has the potential to be used as an immune booster in this pandemic.

Indian Mustard (Brassica juncea L. Czern., Family: Brassicaceae): Indian mustard ('Shorisha' in Bengali language) is a popular condiment in Bangladesh. It is grown as a root and leaf vegetable and as an oilseed. It has phyto-remediating properties (Mazumder et al., 2020). Antiviral activity of this plant against influenza virus was found through invitro studies (Lee et al., 2014). Curcumenol and $\mathrm{N}$ Desmethylselegiline, two constituents of this plant, showed potential to be selective and strong lead compound against COVID-19 in-silico (Dave et al., 2020). Further studies are required to make conclusive decision in this regard.

Licorice (Glycyrrhiza glabra L., Family: Fabaceae): Licorice ('Joshtimodhu' in Bangali language) is a well-known medicinal plant in 
<smiles>CC(C)=CCC/C(C)=C/CC/C(C)=C/CC[C@]1(C)CCc2c(C)c(O)c(C)c(C)c2O1</smiles>

Cocos mucifera (Fitriani et al., 2020)

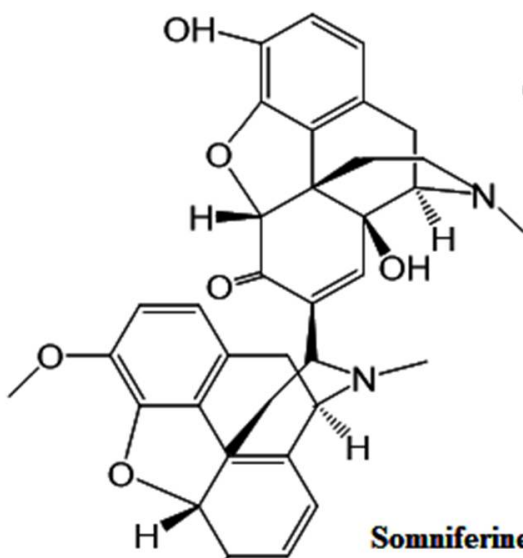

Somniferine

Withania somnifera

(Shree et al., 2020;

Srivastava et al., 2020)

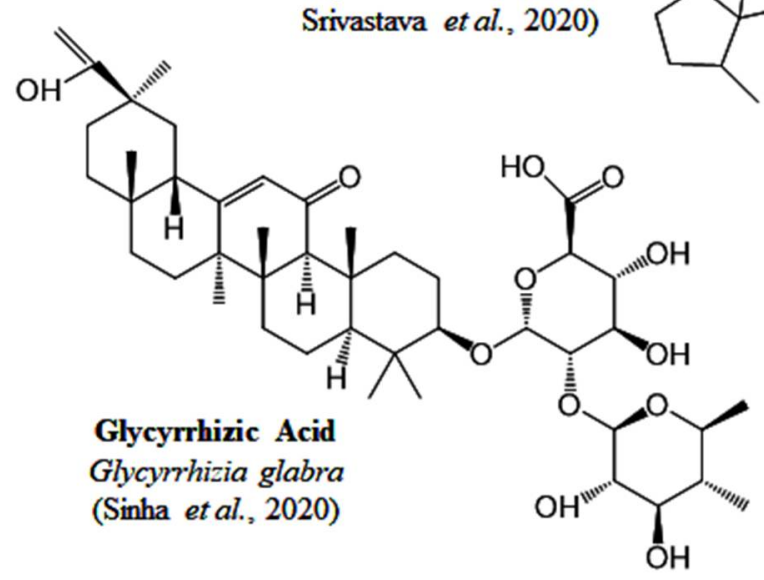<smiles>C#CN[C@H](C)Cc1ccccc1</smiles>

N-desmethylselegiline Brassica juncea

(Dave et al., 2020)

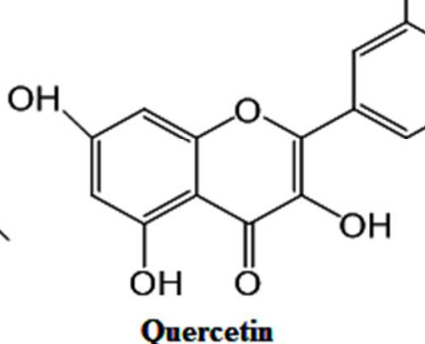
Allium sativum (Khubber et al., 2020; Pandey et al., 2021)<smiles>CC1=CC2(O)CC(=C(C)C)CC3(O2)C(C)CCC13</smiles>

Curcumenol Brassica juncea

(Dave et al., 2020)<smiles>C=CCC=C(C)CC</smiles><smiles>[10BH]</smiles><smiles>CNc1ccc(C2CCn3c2nc2ccccc2c3=O)cc1C(=O)OC</smiles>

(Figure 1 Contd.) 
<smiles>COc1ccc2cc3[n+](cc2c1OC)CCc1cc2c(cc1-3)OCO2</smiles><smiles>COc1cc(/C=C/C(=O)CC(=O)/C=C/c2ccc(O)c(OC(C)(Cl)Cl)c2)ccc1O</smiles>

\section{Allicin}

Allium sativum

(Khubber et al., 2020;

Pandey et al., 2021)

Antony et al., 1999;

Kim et al., 2016)

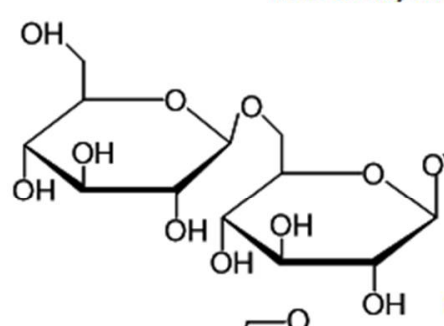<smiles>CCC(C)C(C)(C)C1CCC2(C)C(C)CCC2(C)C1C1CCC2(C)C(C)=C(C)C(=O)OC2C1</smiles><smiles>C=C1CC[C@]2(C(CO)CO)[C@@H](C)CC[C@]2(C)[C@@H](O)CC1CC=C1C(=O)OC[C@H]1O</smiles>

$\mathrm{OH}^{\text {Andrographolide }}$ Andrographis paniculata

(Murugan et al., 2020; Rajagopal et al., 2020; Sukardiman et al., 2020)

Withanoside V

Withania somnifera (Shree et al., 2020; Srivastava et

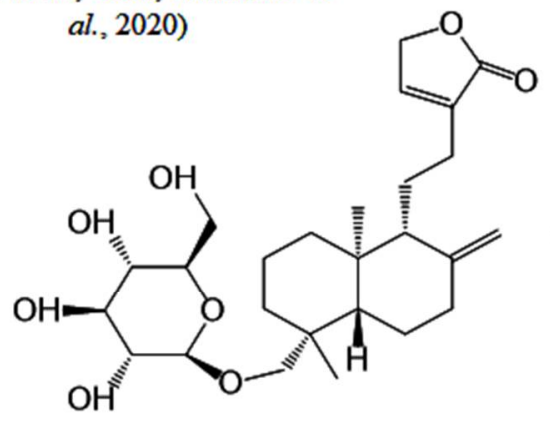

Neoandrographolide Andrographis paniculata (Murugan et al., 2020; Rajagopal et al., 2020; Sukardiman et al., 2020)<smiles>O=c1cc(-c2ccccc2)oc2c([C@@H]3O[C@H](CO)[C@@H](O)[C@H](O)[C@H]3O)c(O)c([C@@H]3O[C@H](CO)[C@@H](O)[C@H](O)[C@H]3O)c(O)c12</smiles>

(Shree et al., 2020; Varshney et al., 2020)<smiles>COc1cc(/C=C/C2=CC(=O)C[C@H](c3ccc(O)c(OC)c3)O2)ccc1O</smiles><smiles>C=CC[S@](C)(=O)C[C@H](N)C(=O)O</smiles>

Allium sativum (Khubber et al., 2020; Pandey et al., 2021)

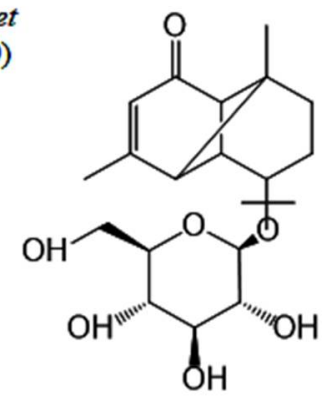

Tinocordiside

Tinospora cordifolia (Chowdhury, 2020; Kumar and Chander, 2020; Shree et al., 2020)

Figure 1. Structures of potential immunostimulating, anti-inflammatory and antiviral phytoconstituents in prospective indigenous plants of Bangladesh 
Bangladesh. Its antiviral, antibiotic and immunomodulatory properties are well-reported. Several constituents of licorice, e.g., glyasperin A and glycyrrhizic acid, displayed strong binding potential to SARS-CoV-2 spike protein and nonstructural proteins (Sinha et al., 2020). Its use in COVID-19 may be advantageous.

Malabar nut (Justicia adhatoda L., Family: Acanthaceae): Malabar nut ('Basok' in Bengali language) is a widely-used medicinal plant. Its leaves have been reported to contain alkaloids and other phytochemicals which were found to have antiviral, antibacterial, and immunomodulatory activities. Such an alkaloid, anisotine, was found to be potentially active in-silico against SARS-CoV-2 protease enzymes (Ghosh et al., 2021). Besides, in-vitro studies reported that its extracts were effective against influenza virus (Chavan and Chowdhary, 2014). This plant may be a potentially beneficial immune booster in this pandemic.

Moringa (Moringa oleifera Lam., Family: Moringaceae): Moringa ('Shojina' in Bengali language) is a common vegetable in Bangladesh. It has been reported to display anti-inflammatory, antioxiadent, antiviral, and anticancer activities. It is also an immune booster. Studies showed that hand sanitizers made by using its leaves could help prevent the transmission of COVID-19 (Arifan et al., 2021). In-silico studies found that its compounds, e.g., pterygospermin, quercetin, and kaempferol, might work against SARS-CoV-2 (Fitriani et al., 2020; Shaji, 2020). Regular intake of moringa may be advantageous.

Neem (Azadirachta indica A. Juss., Family: Meliaceae): Neem ('Neem' in Bengali language), alternatively known as neem or Indian lilac, possesses incredible antiviral activity. The leaf extract of neem was previously proven active against coxsackievirus B-4 due to the possession of azadirachtin. Thus, it might act as an immune booster against COVID-19 (Das et al., 2020).

Onion (Allium cepa L., Family: Amaryllidaceae): Onion ('Piyaj' in Bengali language) is a widely used condiment in Bangladesh. It has been reported to have antiviral activity in several studies. Phytoconstituents of onion, e.g., alliin, oleanolic acid, and S-propyl cysteine, showed virus protein and genetic material blocking activity. In-silico studies also found its potential against COVID-19 virus (Fitriani et al., 2020; Pandey et al., 2021). Regular use of onion may be useful against COVID-19 through this immune booster property.

Pomegranate (Punica granatum L., Family: Lythraceae): Pomegranate ('Anar' in Bengali language) fruit juice possesses antimicrobial, antifungal, antiviral, antioxidant,immunity-boosting, and anti-carcinogenic properties (Bassiri-Jahromi, 2018; Karimi et al, 2017; Ricci et al, 2006; Singh et al., 2002). The fruit contain high amount of vitamin $\mathrm{C}$ and $\mathrm{E}$ which can defend us from severe infections by augmenting the production of antibodies in our body (Baidya and Sethy, 2020). Unani scholars are prescribing this fruit juice in the treatment of COVID-19, since they asserted that it has cleansing effect on body humors (Nikhat and Fazil, 2020).

Tamarind (Tamarindus indica L., Family: Fabaceae): Tamarind ('Tetul' in Bengali language) plants grow naturally and are also cultivated in tropical and sub-tropical regions of the world for their fruits. For centuries, the pulp of the fruit is used to prepare beverages, confectionaries and also widely used as traditional medicine in treating different ailments. The polyphenols and tannins in tamarind have antioxidant, anti-inflammatory and analgesic properties (Siddhuraju, 2007). Furthermore, they contain vitamin $\mathrm{C}$ which can potentiate the immune system as well as prevent microbial and fungal infections (Deet al., 2009).In 1993, Sreelekha, et al., isolated a polysaccharide from $T$. indica which exhibited immunomodulatory properties like phagocytic enhancement and inhibition of leukocyte migration during cell proliferation (Sreelekha et al., 1993). The pulp of the fruit is being prescribed by unani practitioners as health protective drug in COVID-19 epidemic (Nikhat and Fazil, 2020).

Tea (Camellia sinensis L. Kuntze, Family: Theaceae): Tea ('Cha' in Bengali language) can provide very proficient immunomodulatory and 
Table 1. Medicinal plants/Natural products of Bangladesh with reported anti-inflammatory, antiviral, immunostimulanting, and respiratory distress syndrome improvement activities

\begin{tabular}{|c|c|c|c|c|}
\hline $\begin{array}{l}\text { Name (Bangla } \\
\text { Name) }\end{array}$ & $\begin{array}{l}\text { Scientific Name } \\
\text { (Family) }\end{array}$ & Test sample & Activity & References \\
\hline \multirow{7}{*}{$\begin{array}{l}\text { Basil } \\
\text { (Tulsi) }\end{array}$} & \multirow{7}{*}{$\begin{array}{l}\text { Ocimum sanctum } \mathrm{L} . \\
\text { (Lamiaceae) }\end{array}$} & Fixed oils & Anti-inflammatory & Singh (1998) \\
\hline & & Aqueous extract & Anti-inflammatory & $\begin{array}{l}\text { Reshma and } \\
\text { Brindha (2014) }\end{array}$ \\
\hline & & $\begin{array}{l}\text { Terpenoid and polyphenol } \\
\text { extracts from leaves }\end{array}$ & Antiviral & Ghoke et al. (2018) \\
\hline & & Aqueous extract & Immunostimulant & Jeba et al. (2011) \\
\hline & & Seed oil & Immunostimulant & $\begin{array}{l}\text { Mediratta et al. } \\
\text { (2002) }\end{array}$ \\
\hline & & Fixed oil & Resist respiratory tract infection & Saini et al. (2009) \\
\hline & & $\begin{array}{l}\text { Vicenin, ursolic acid, tulsinol } \\
\text { A-G and isorientin } 4^{\prime}-O- \\
\text { glucoside } 2^{\prime \prime}-O-p- \\
\text { hydroxybenzoate }\end{array}$ & $\begin{array}{l}\text { Showed strong inhibiting property } \\
\text { against SARS-cov-2 virus in-silico }\end{array}$ & $\begin{array}{l}\text { Shree et al. (2020); } \\
\text { Varshney et al. } \\
(2020)\end{array}$ \\
\hline \multirow{7}{*}{$\begin{array}{l}\text { Black cumin } \\
\text { (Kalojira) }\end{array}$} & \multirow{7}{*}{$\begin{array}{l}\text { Nigella sativa } \mathrm{L} . \\
\text { (Ranunculaceae) }\end{array}$} & Aqueous extract & Anti-inflammatory & Al-Ghamdi (2001) \\
\hline & & Seed polyphenols & Anti-inflammatory & $\begin{array}{l}\text { Ghannadi et al. } \\
\text { (2005) }\end{array}$ \\
\hline & & Aqueous extract & Immunostimulant & $\begin{array}{l}\text { Boskabady et al. } \\
\text { (2011) }\end{array}$ \\
\hline & & Ethanolic extract & Polyclonal IgM production & Sarker et al. (2011) \\
\hline & & Volatile oil & $\begin{array}{l}\text { Increases the respiratory rate and } \\
\text { the intratracheal pressure }\end{array}$ & $\begin{array}{l}\text { El Tahir et al. } \\
\text { (1993) }\end{array}$ \\
\hline & & $\begin{array}{l}\text { Nigelledine, hederagenin, and } \\
\alpha \text {-hederin. }\end{array}$ & $\begin{array}{l}\text { Moderate anti-covid-19 property } \\
\text { found according to in-silico study }\end{array}$ & $\begin{array}{l}\text { Koshak and Koshak } \\
(2020)\end{array}$ \\
\hline & & Seeds & $\begin{array}{l}\text { Zinc supplement efficiency in } \\
\text { managing covid- } 19 \text { might be } \\
\text { enhanced by black cumin }\end{array}$ & Rahman (2020) \\
\hline \multirow{3}{*}{$\begin{array}{l}\text { Black } \\
\text { mulberry } \\
\text { (Toot) }\end{array}$} & \multirow[t]{3}{*}{$\begin{array}{l}\text { Morus nigra L. } \\
\text { (Moraceae) }\end{array}$} & $\begin{array}{l}\text { Total flavonoids extract from } \\
\text { fruit powder }\end{array}$ & Anti-inflammatory & Chen et al. (2016) \\
\hline & & $\begin{array}{l}\text { Morniga M (mannose-specific } \\
\text { lectin) from the bark }\end{array}$ & Antiviral & $\begin{array}{l}\text { Keyaerts et al. } \\
(2007)\end{array}$ \\
\hline & & $\begin{array}{l}\text { Crude root bark extract, } \\
\text { isolated compounds (Diels- } \\
\text { Alder-type adduct) from the } \\
\text { root bark extract }\end{array}$ & Anti-tuberculosis activity & $\begin{array}{l}\text { Mascarello et al. } \\
\text { (2018) }\end{array}$ \\
\hline \multirow{6}{*}{$\begin{array}{l}\text { Black pepper } \\
\text { (Gol Morich) }\end{array}$} & \multirow{6}{*}{$\begin{array}{l}\text { Piper nigrum L. } \\
\text { (Piperaceae) }\end{array}$} & Essential oil & Anti-inflammatory & Jeena et al. (2014) \\
\hline & & $\begin{array}{l}\text { Alkaloids isolated from fruit } \\
\text { extract }\end{array}$ & Anti-inflammatory & Pei et al. (2020) \\
\hline & & $\begin{array}{l}\text { Piperamides isolated from fruit } \\
\text { extract and piperine alkaloid } \\
\text { extracts from seeds }\end{array}$ & Antiviral & Mair et al. (2016) \\
\hline & & Fruit extract & Immunostimulant & $\begin{array}{l}\text { Sunila and Kuttan } \\
(2004) \text {; Sarker } \\
(2012)\end{array}$ \\
\hline & & \multirow[t]{2}{*}{ Piperine (isolated compound) } & Anti-asthmatic & Kim and Lee (2009) \\
\hline & & & $\begin{array}{l}\text { Higher binding potential with the } \\
\text { virus nucleocapsid and subsequent } \\
\text { decrease of the virulence seen as } \\
\text { per in-silico studies }\end{array}$ & $\begin{array}{l}\text { Choudhary et al. } \\
\text { (2020) }\end{array}$ \\
\hline
\end{tabular}




\begin{tabular}{|c|c|c|c|c|}
\hline $\begin{array}{l}\text { Name (Bangla } \\
\text { Name) }\end{array}$ & $\begin{array}{l}\text { Scientific Name } \\
\text { (Family) }\end{array}$ & Test sample & Activity & References \\
\hline \multirow{5}{*}{$\begin{array}{l}\text { Caraway } \\
\text { (Mouri) }\end{array}$} & \multirow{5}{*}{$\begin{array}{l}\text { Trachyspermum } \\
\text { ammi (L.) Sprague } \\
\text { (Apiaceae) }\end{array}$} & Aquo-alcoholic seed extract & Anti-inflammatory & Umar et al. (2012) \\
\hline & & Essential oil & Antiviral & Roy et al. (2015) \\
\hline & & $\begin{array}{l}n \text {-hexane, chloroform, and } \\
\text { methanol seed extract }\end{array}$ & Immunostimulant & Siddiqui et al. (2019) \\
\hline & & $\begin{array}{l}\text { Novel glycoprotein isolated } \\
\text { from aqueous extract }\end{array}$ & Immunostimulant & Shruthi et al. (2017) \\
\hline & & Essential oil & $\begin{array}{l}\text { Antibacterial activity against } \\
\text { bacterial pneumonia }\end{array}$ & Mehdi et al. (2014) \\
\hline \multirow{4}{*}{$\begin{array}{l}\text { Chebulic } \\
\text { Myrobalan } \\
\text { (Haritaki) }\end{array}$} & \multirow{4}{*}{$\begin{array}{l}\text { Terminalia chebula } \\
\text { Retz. } \\
\text { (Combretaceae) }\end{array}$} & Hydro-alcoholic fruit extract & Anti-inflammatory & Bag et al. (2013) \\
\hline & & $\begin{array}{l}\text { Fruit extract, chebulagic acid } \\
\text { and chebulinic acid }\end{array}$ & Antiviral & Kesharwani et al. (2017) \\
\hline & & Fruit extract & Immunostimulant & $\begin{array}{l}\text { Haque et al. (2021); } \\
\text { Shivaprasad } \text { et al. (2006) }\end{array}$ \\
\hline & & $\begin{array}{l}\text { Water-Extracted } \\
\text { Polysaccharide from fruit }\end{array}$ & Antitussive & Nosalova et al. (2013) \\
\hline \multirow[t]{4}{*}{$\begin{array}{l}\text { Citron } \\
\text { (Jamir Lebu) }\end{array}$} & \multirow[t]{4}{*}{$\begin{array}{l}\text { Citrus medica L. } \\
\text { (Rutaceae) }\end{array}$} & $\begin{array}{l}\text { Fruit extract, flower extract, } \\
\text { leaves extract }\end{array}$ & Anti-inflammatory & Menichini et al. (2011) \\
\hline & & $\begin{array}{l}\text { Rhoifolin, Naringin, } \\
\text { Neohesperidin, Apigenin 6,8- } \\
\text { di-C-glucoside, Hesperidin, } \\
\text { Xanthin }\end{array}$ & Antiviral & Haridas et al. (2021) \\
\hline & & Fermented fruit extract & Immunostimulant & Huber et al. (2012) \\
\hline & & $\begin{array}{l}\text { Fruit juice, ethanolic extracts } \\
\text { of root, leaf, bark, peel and } \\
\text { pulp }\end{array}$ & $\begin{array}{l}\text { Antimicrobial activity } \\
\text { against bacterial pneumonia } \\
\text { pathogen }\end{array}$ & Sah et al. (2011) \\
\hline \multirow{5}{*}{$\begin{array}{l}\text { Clove } \\
\text { (Labongo) }\end{array}$} & \multirow{5}{*}{$\begin{array}{l}\text { Syzygium } \\
\text { aromaticum ((L.) } \\
\text { Merr. } \\
\text { (Myrtaceae) }\end{array}$} & Flower bud extract & Anti-inflammatory & Tanko et al. (2008) \\
\hline & & Eugeniin & Antiviral & Kurokawa et al. (1998) \\
\hline & & $\begin{array}{l}\text { Novel green silver } \\
\text { nanoparticles produced using } \\
\text { aqueous buds extract }\end{array}$ & Antiviral & Mehmood et al. (2020) \\
\hline & & Clove extract and essential oil & Immunostimulant & Bachiega (2012) \\
\hline & & Essential oil & $\begin{array}{l}\text { Antibacterial activity in } \\
\text { respiratory infections }\end{array}$ & Inouye et al. (2001) \\
\hline \multirow{5}{*}{$\begin{array}{l}\text { Coconut } \\
\text { (Narkel) }\end{array}$} & \multirow{5}{*}{$\begin{array}{l}\text { Cocos nucifera } \mathrm{L} . \\
\text { (Arecaceae) }\end{array}$} & Crude extract & Anti-inflammatory & Rinaldi et al. (2009) \\
\hline & & $\begin{array}{l}\text { Crude extract, polyphenolics } \\
\text { from husk fiber extract }\end{array}$ & Antiviral & Esquenazi et al. (2002) \\
\hline & & Coconut protein & Immunostimulant & $\begin{array}{l}\text { Vigila and Baskaran } \\
\text { (2008) }\end{array}$ \\
\hline & & $\begin{array}{l}\text { Synthesized silver } \\
\text { nanoparticles using } \\
\text { inflorescence extract }\end{array}$ & Antibacterial activity & Mariselvamet al. (2014) \\
\hline & & $\begin{array}{l}\alpha \text {-tocotrienol from coconut } \\
\text { fruit }\end{array}$ & $\begin{array}{l}\text { In-silico study displayed anti- } \\
\text { covid-19 activity }\end{array}$ & Fitriani et al. (2020) \\
\hline \multirow{6}{*}{$\begin{array}{l}\text { Creat } \\
\text { (Kalomegh or } \\
\text { Chirotta) }\end{array}$} & \multirow{6}{*}{$\begin{array}{l}\text { Andrographis } \\
\text { paniculata } \\
\text { (Bum.f.) Nees } \\
\text { (Acanthaceae) }\end{array}$} & Whole plant extract & Anti-inflammatory & Sheeja et al. (2006) \\
\hline & & $\begin{array}{l}\text { Crude extract, Ent-labdene } \\
\text { Diterpenes isolated from } \\
\text { extract }\end{array}$ & Antiviral & Wiart et al. (2005) \\
\hline & & Ethanolic extract & Antiviral & Churiyah et al. (2015) \\
\hline & & Ethanolic extract & Immunostimulant & Churiyah et al. (2015) \\
\hline & & $\begin{array}{l}\text { Crude drug, standardized } \\
\text { extract }\end{array}$ & $\begin{array}{l}\text { Alleviates symptoms of } \\
\text { uncomplicated upper } \\
\text { respiratory tract infection } \\
\text { (urti) }\end{array}$ & Coon and Ernst (2004) \\
\hline & & $\begin{array}{l}\text { Dihydroxy dimethoxy flavone, } \\
\text { andrographolide, and } \\
\text { neoandrographolide }\end{array}$ & $\begin{array}{l}\text { Showed strong binding } \\
\text { affinity for the sars-cov- } 2 \\
\text { virus active site in-silico }\end{array}$ & $\begin{array}{l}\text { Murugan } \text { et al. }(2020) \text {; } \\
\text { Rajagopal } \text { et al. }(2020) \text {; } \\
\text { Sukardiman } \text { et al. }(2020)\end{array}$ \\
\hline
\end{tabular}




\begin{tabular}{|c|c|c|c|c|}
\hline $\begin{array}{l}\text { Name (Bangla } \\
\text { Name) }\end{array}$ & $\begin{array}{l}\text { Scientific Name } \\
\text { (Family) }\end{array}$ & Test sample & Activity & References \\
\hline \multirow[t]{4}{*}{ Neem } & \multirow{4}{*}{$\begin{array}{l}\text { Azadirachta indica } \\
\text { A. Juss. } \\
\text { (Meliaceae) }\end{array}$} & Leaves and bark extract & Anti-inflammatory & $\begin{array}{l}\text { Okpanyi and Ezeukwu } \\
\text { (1981) }\end{array}$ \\
\hline & & $\begin{array}{l}\text { Polysaccharides from leaves } \\
\text { extract }\end{array}$ & Antiviral & $\begin{array}{l}\text { Faccin-Galhardi et al. } \\
\text { (2012) }\end{array}$ \\
\hline & & Neem oil & Immunostimulant & Upadhyay et al. (1992) \\
\hline & & Leaf extract & $\begin{array}{l}\text { Protective effects against } \\
\text { pulmonary inflammation }\end{array}$ & Lee et al. (2017) \\
\hline \multirow[t]{5}{*}{$\begin{array}{l}\text { Onion } \\
\text { (Piyaj) }\end{array}$} & \multirow[t]{5}{*}{$\begin{array}{l}\text { Allium cepa } \mathrm{L} . \\
\text { (Amaryllidaceae) }\end{array}$} & $\begin{array}{l}\text { Volatile sample extracted from } \\
\text { freeze-dried onion sprout }\end{array}$ & Anti-inflammatory & Takahashi (2008) \\
\hline & & Essential oil & Antiviral & Romeilah et al. (2010) \\
\hline & & Scale Extract & Immunostimulant & Elberry et al. (2014) \\
\hline & & Methanolic extract & Anti-asthmatic & Oliveira et al. (2015) \\
\hline & & Fleshy edible part of the plant & $\begin{array}{l}\text { Prevents carbon monoxide- } \\
\text { induced lung tissue toxicity }\end{array}$ & Ogadinma et al. (2018) \\
\hline \multirow[t]{4}{*}{$\begin{array}{l}\text { Pomegranate } \\
\text { (Anar) }\end{array}$} & \multirow[t]{4}{*}{$\begin{array}{l}\text { Punica granatum L. } \\
\text { (Lythraceae) }\end{array}$} & $\begin{array}{l}\text { Purified pomegranate } \\
\text { polyphenol extract }\end{array}$ & Antiviral & Haidari et al. (2009) \\
\hline & & Peel extract & Immunostimulant & Labsi et al. (2016) \\
\hline & & Leaf extract & $\begin{array}{l}\text { Anti-inflammatory effects on } \\
\text { acute lung inflammation }\end{array}$ & $\begin{array}{l}\text { Costa Rodrigues Pinheiro } \\
\text { (2018) }\end{array}$ \\
\hline & & Leaves extract & $\begin{array}{l}\text { In-vitro anti-cancer effect in } \\
\text { lungs }\end{array}$ & Li et al. (2016) \\
\hline \multirow{4}{*}{$\begin{array}{l}\text { Tamarind } \\
\text { (Tetul) }\end{array}$} & \multirow{4}{*}{$\begin{array}{l}\text { Tamarindus indica } \\
\text { L. } \\
\text { (Fabaceae) }\end{array}$} & Seeds & Anti-inflammatory & Suralkar et al. (2012) \\
\hline & & Stem bark extract & Antiviral & Okoh et al. (2017) \\
\hline & & $\begin{array}{l}\text { Polysaccharide pst001 isolated } \\
\text { from the seed kernel }\end{array}$ & Immunostimulant & Aravind et al. (2012) \\
\hline & & Leaf extract & Anti-asthmatic activity & Tayade et al. (2009) \\
\hline \multirow{5}{*}{$\begin{array}{l}\text { Flaxseed } \\
\text { (Tishi) }\end{array}$} & \multirow{5}{*}{$\begin{array}{l}\text { Linum usitatissimum } \\
\mathrm{L} . \\
\text { (Linaceae) }\end{array}$} & Fixed oil & Anti-inflammatory & Kaithwas et al. (2011) \\
\hline & & Plant extract & Antiviral & Mouhajir et al. (2001) \\
\hline & & n-butanol fraction of flaxseed & Immunostimulant & Kasote et al. (2012) \\
\hline & & Flaxseed oil & Immunostimulant & Gaber and Badawy (2019) \\
\hline & & Flax seeds derived lignan & $\begin{array}{l}\text { Reduction of oxidative lung } \\
\text { damage and decreased lung } \\
\text { fibrosis }\end{array}$ & Lee et al. (2009) \\
\hline \multirow{11}{*}{$\begin{array}{l}\text { Garlic } \\
\text { (Roshun) }\end{array}$} & \multirow{11}{*}{$\begin{array}{l}\text { Allium sativum } \mathrm{L} . \\
\text { (Amaryllidaceae) }\end{array}$} & Garlic oil & Anti-inflammatory & Hussein et al. (2017) \\
\hline & & Thiacremone & Anti-inflammatory & Hussein et al. (2017) \\
\hline & & Aged garlic extract & Immunostimulant & Londhe (2011) \\
\hline & & Garlic extract & Anti-inflammatory & Weber et al. (1992) \\
\hline & & Garlic extract & Antiviral & $\begin{array}{l}\text { Meléndez-Villanueva } \\
\text { et al. (2019) }\end{array}$ \\
\hline & & $\begin{array}{l}\text { Aqueous extract in gold } \\
\text { nanoparticles }\end{array}$ & Antiviral & Shojai et al. (2016) \\
\hline & & Aqueous extract & Antiviral & Choi (2018) \\
\hline & & Garlic oil & Antiviral & Hussein et al. (2017) \\
\hline & & Raw garlic extract & Immunostimulant & Londhe (2011) \\
\hline & & Diallyl disulfide & $\begin{array}{l}\text { Induction of apoptosis in } \\
\text { human non-small cell lung } \\
\text { carcinoma h1299 cells }\end{array}$ & Cao et al. (2008) \\
\hline & & Quercetin, allicin and alliin & $\begin{array}{l}\text { In-silico promising activity } \\
\text { against covid-19 }\end{array}$ & $\begin{array}{l}\text { Khubber et al. (2020); } \\
\text { Pandey et al. }(2021)\end{array}$ \\
\hline
\end{tabular}




\begin{tabular}{|c|c|c|c|c|}
\hline $\begin{array}{l}\text { Name (Bangla } \\
\text { Name) }\end{array}$ & $\begin{array}{l}\text { Scientific Name } \\
\text { (Family) }\end{array}$ & Test sample & Activity & References \\
\hline \multirow[t]{8}{*}{$\begin{array}{l}\text { Giloy } \\
\text { (Guloncho) }\end{array}$} & \multirow{8}{*}{$\begin{array}{l}\text { Tinospora cordifolia } \\
\text { (Thunb.) Miers } \\
\text { (Menispermaceae) }\end{array}$} & Aqueous extract & Anti-inflammatory & Patgiri et al. (2014) \\
\hline & & Aqueous extract & Anti-inflammatory & Birla et al. (2019) \\
\hline & & Root extract & Antiviral & Mittal et al. (2014) \\
\hline & & $\begin{array}{l}\text { Ethanol and petroleum ether } \\
\text { extract }\end{array}$ & Immunostimulant & Sudhakaran et al. (2006) \\
\hline & & Sedimented aqueous extract & Immunostimulant & Bishayi et al. (2002) \\
\hline & & Leaf extract & $\begin{array}{l}\text { Toxic against human lung } \\
\text { adenocarcinoma cell line } \\
\text { a549 }\end{array}$ & Mittal et al. (2020) \\
\hline & & Aqueous ethanolic extract & $\begin{array}{l}\text { Decreased airway hyper- } \\
\text { responsiveness, anti- } \\
\text { asthmatic }\end{array}$ & Tiwari et al. (2014) \\
\hline & & Berberine and tinocordiside & $\begin{array}{l}\text { Showed potential for } \\
\text { inhibition of viral replication } \\
\text { of sars-cov-2 in-silico } \\
\text { through modulating viral } \\
\text { protease activity }\end{array}$ & $\begin{array}{l}\text { Chowdhury (2020); Kumar } \\
\text { and Chander (2020); Shree } \\
\text { et al. (2020) }\end{array}$ \\
\hline \multirow{7}{*}{$\begin{array}{l}\text { Ginger } \\
\text { (Ada) }\end{array}$} & \multirow{7}{*}{$\begin{array}{l}\text { Zingiber officinale } \\
\text { Roscoe } \\
\text { (Zingiberaceae) }\end{array}$} & Fresh rhizomes & Anti-inflammatory & Mahluji et al. (2013) \\
\hline & & Hydro-alcoholic extract & Anti-inflammatory & Penna et al. (2003) \\
\hline & & $\begin{array}{l}\text { Hot water extracts of fresh } \\
\text { ginger }\end{array}$ & Antiviral & Chang et al. (2013) \\
\hline & & Aquatic plant extract & Antiviral & Kaushik et al. (2020) \\
\hline & & Rhizome & Immunostimulant & $\begin{array}{l}\text { Amri and Touil-Boukoffa } \\
\text { (2016) }\end{array}$ \\
\hline & & Essential oil & Immunostimulant & Carrasco et al. (2009) \\
\hline & & $\begin{array}{l}\text { Hydro-alcoholic extract of } \\
\text { rhizome }\end{array}$ & $\begin{array}{l}\text { Attenuated rat trachea } \\
\text { hyperreactivity (rthr) and } \\
\text { exerted anti-inflammatory } \\
\text { effect on lung inflammation }\end{array}$ & Aimbire et al. (2007) \\
\hline \multirow[t]{2}{*}{$\begin{array}{l}\text { Golden } \\
\text { shower tree }\end{array}$} & \multirow[t]{4}{*}{$\begin{array}{l}\text { Cassia fistula } \mathrm{L} . \\
\text { (Fabaceae) }\end{array}$} & $\begin{array}{l}\text { Aqueous and methanolic } \\
\text { extracts }\end{array}$ & Anti-inflammatory & $\begin{array}{l}\text { Ilavarasan and } \\
\text { Venkataraman (2005) }\end{array}$ \\
\hline & & Rhein & Anti-inflammatory & Antonisamy et al. (2019) \\
\hline \multirow[t]{2}{*}{ (Shonalu) } & & Callus culture & Antiviral & Arora et al. (2016) \\
\hline & & $\begin{array}{l}\text { Hot aqueous extract of pods } \\
\text { and leaves }\end{array}$ & Immunostimulant & Laxmi et al. (2015) \\
\hline \multirow{4}{*}{$\begin{array}{c}\text { Gymnema } \\
\text { (Gurmar) }\end{array}$} & \multirow{4}{*}{$\begin{array}{l}\text { Gymnema sylvestre } \\
\mathrm{R} \text {. Br. } \\
\text { (Apocyanaceae) }\end{array}$} & Aqueous extract of leaves & Anti-inflammatory & Diwan et al. (1995) \\
\hline & & Methanolic extract & $\begin{array}{l}\text { Anti-inflammatory, } \\
\text { Immunostimulant }\end{array}$ & Khan et al. (2019) \\
\hline & & Gymnemagenol & Antiviral & Khanna et al. (2011) \\
\hline & & Methanolic extract of leaf & Immunostimulant & Singh et al. (2015) \\
\hline \multirow{11}{*}{$\begin{array}{l}\text { Indian } \\
\text { Ginseng } \\
\text { (Ashwagandh } \\
\text { a) }\end{array}$} & \multirow{11}{*}{$\begin{array}{l}\text { Withania somnifera } \\
\text { (L.) Dunal } \\
\text { (Solanaceae) }\end{array}$} & Root powder & Anti-inflammatory & Gupta and Singh (2014) \\
\hline & & Hydro-alcoholic extract & Anti-inflammatory & Chandra et al. (2012) \\
\hline & & Hydro-alcoholic root extract & Antiviral & Pant et al. (2012) \\
\hline & & Water extract from leaves & Antiviral & Mofed et al. (2020) \\
\hline & & Withaferin A & Antiviral & Cai et al. (2015) \\
\hline & & Ethanolic extract & Immunostimulant & Harikrishnan et al. (2012) \\
\hline & & Root extract & Immunostimulant & Zhou and Zhang (2008) \\
\hline & & $\begin{array}{l}\text { Bioactive fraction-withanolide } \\
\text { D }\end{array}$ & $\begin{array}{l}\text { Significantly inhibited the } \\
\text { metastatic colony formation } \\
\text { of the melanoma in lungs }\end{array}$ & Leyon and Kuttan (2004) \\
\hline & & Root powder & $\begin{array}{l}\text { Tumor-preventing activity } \\
\text { against } \\
\text { Urethane-induced lung } \\
\text { adenomas }\end{array}$ & Singh et al. (1986) \\
\hline & & Root powder & $\begin{array}{l}\text { Protective effect in } \\
\text { monocrotaline-induced } \\
\text { pulmonary hypertension }\end{array}$ & Kaur et al. (2015) \\
\hline & & $\begin{array}{l}\text { Somniferine and withanoside } \\
\mathrm{V}\end{array}$ & $\begin{array}{l}\text { In-silico studies displayed } \\
\text { promising inhibition of } \\
\text { covid-19. }\end{array}$ & $\begin{array}{l}\text { Shree et al. (2020); } \\
\text { Srivastava } \text { et al. }(2020)\end{array}$ \\
\hline
\end{tabular}




\begin{tabular}{|c|c|c|c|c|}
\hline $\begin{array}{l}\text { Name (Bangla } \\
\text { Name) }\end{array}$ & $\begin{array}{l}\text { Scientific Name } \\
\text { (Family) }\end{array}$ & Test sample & Activity & References \\
\hline $\begin{array}{l}\text { Indian } \\
\text { mustard }\end{array}$ & Brassica juncea (L.) & $\begin{array}{l}\text { Petroleum ether and ethanolic } \\
\text { extracts }\end{array}$ & Anti-inflammatory & Sindhoor et al. (2012) \\
\hline \multirow[t]{4}{*}{ (Shorisha) } & \multirow[t]{4}{*}{ (Brassicaceae) } & Methanolic seeds extract & Anti-inflammatory & Chouhan et al. (2014) \\
\hline & & Subcritical water extract & Antiviral & Lee et al. (2014) \\
\hline & & Ethanol extract & $\begin{array}{l}\text { In-vitro anti-cancer activities } \\
\text { against lung cancers }\end{array}$ & Kwak et al. (2016) \\
\hline & & $\begin{array}{l}\text { Curcumenol and } N \text { - } \\
\text { desmethylselegiline }\end{array}$ & $\begin{array}{l}\text { In-silico studies displayed } \\
\text { promising inhibition of } \\
\text { COVID-19 }\end{array}$ & Dave et al. (2020) \\
\hline Licorice & $\begin{array}{l}\text { Glycyrrhiza glabra } \\
\text { L. }\end{array}$ & Hydro-alcoholic extract & Anti-inflammatory & $\begin{array}{l}\text { Nirmala and Selvaraj } \\
\text { (2011) }\end{array}$ \\
\hline \multirow[t]{9}{*}{ (Joshtimodhu) } & \multirow[t]{9}{*}{ (Fabaceae) } & Leaf extracts & Anti-inflammatory & Frattaruolo et al. (2019) \\
\hline & & Food & Antiviral & Pastorino et al. (2018) \\
\hline & & Glycyrrhetinic acid & Antiviral & Pastorino et al. (2018) \\
\hline & & $\begin{array}{l}\text { Aqueous liquorice extract and } \\
\text { its combination with zinc }\end{array}$ & Immunostimulant & Mazumder et al. (2012) \\
\hline & & Aqueous methanolic extract & Immunostimulant & Hussain et al. (2017) \\
\hline & & Aquo-alcoholic extract & $\begin{array}{l}\text { Effective against lung } \\
\text { infection caused by } \\
\text { pseudomonas aeruginosa }\end{array}$ & Chakotiya et al. (2017) \\
\hline & & $\begin{array}{l}\text { Glycyrrhizin extracted from } \\
\text { root }\end{array}$ & $\begin{array}{l}\text { Reduced the development of } \\
\text { acute lung inflammation } \\
\text { (pleurisy) }\end{array}$ & Menegazzi et al. (2008) \\
\hline & & Glycyrrhizin & $\begin{array}{l}\text { Anti-inflammatory and } \\
\text { protective effects in } \\
\text { pulmonary inflammation }\end{array}$ & Lee et al. (2019) \\
\hline & & $\begin{array}{l}\text { Glyasperin A and glycyrrhizic } \\
\text { acid }\end{array}$ & $\begin{array}{l}\text { Displayed strong binding } \\
\text { potential to SARS-cov-2 } \\
\text { spike protein and non- } \\
\text { structural proteins in-silico }\end{array}$ & Sinha et al. (2020) \\
\hline \multirow{8}{*}{$\begin{array}{l}\text { Malabar nut } \\
\text { (Basok) }\end{array}$} & \multirow{8}{*}{$\begin{array}{l}\text { Justicia adhatoda } \mathrm{L} . \\
\text { (Acanthaceae) }\end{array}$} & Aqueous extracts from leaves & Anti-inflammatory & Kaur et al. (2013) \\
\hline & & Ethanolic extract of roots & Anti-inflammatory & Mulla et al. (2010) \\
\hline & & Aqueous extract & Antiviral & $\begin{array}{l}\text { Chavan and Chowdhary } \\
\text { (2014) }\end{array}$ \\
\hline & & Methanolic extract & Antiviral & $\begin{array}{l}\text { Chavan and Chowdhary } \\
\text { (2014) }\end{array}$ \\
\hline & & Aqueous extract & Antiviral & Wilson et al. (2021) \\
\hline & & $\begin{array}{l}\text { Methanolic, chloroform and } \\
\text { diethyl extracts of leaves }\end{array}$ & Immunostimulant & $\begin{array}{l}\text { Vinothapooshan and } \\
\text { Sundar (2011) }\end{array}$ \\
\hline & & Leaf extract & $\begin{array}{l}\text { Cytotoxic effect on human } \\
\text { lung carcinoma cell line }\end{array}$ & Latha et al. (2018) \\
\hline & & Anisotine & $\begin{array}{l}\text { Found to be potentially } \\
\text { active } \text { in-silico against sars- } \\
\text { cov- } 2 \text { protease enzymes }\end{array}$ & Ghosh et al. (2020) \\
\hline
\end{tabular}




\begin{tabular}{|c|c|c|c|c|}
\hline $\begin{array}{l}\text { Name (Bangla } \\
\text { Name) }\end{array}$ & $\begin{array}{l}\text { Scientific Name } \\
\text { (Family) }\end{array}$ & Test sample & Activity & References \\
\hline \multirow{17}{*}{$\begin{array}{l}\text { Moringa } \\
\text { (Shojina) }\end{array}$} & \multirow{17}{*}{$\begin{array}{l}\text { Moringa oleifera } \\
\text { Lam. } \\
\text { (Moringaceae) }\end{array}$} & Aqueous root extract & Anti-inflammatory & Ndiaye et al. (2002) \\
\hline & & Aqueous extract & Anti-inflammatory & Rao et al. (1999) \\
\hline & & $\begin{array}{l}\text { Alcoholic extract of the dry } \\
\text { pulverized flowers }\end{array}$ & Anti-inflammatory & Alhakmani et al. (2013) \\
\hline & & Ethanolic extract & Anti-inflammatory & Usman and Barhate (2012) \\
\hline & & Petroleum ether extract & Anti-inflammatory & Usman and Barhate (2012) \\
\hline & & Distilled water extract & Anti-inflammatory & Usman and Barhate (2012) \\
\hline & & Chloroform extract & Anti-inflammatory & Usman and Barhate (2012) \\
\hline & & Aqueous seed extract & Antiviral & Chollom et al. (2012) \\
\hline & & Seeds & Antiviral & Xiong et al. (2021) \\
\hline & & Leaves aqueous extract & Antiviral & Nasr-Eldin et al. (2017) \\
\hline & & Leaf extract & Antiviral & Younus et al. (2016) \\
\hline & & $\begin{array}{l}\text { Hot aqueous and ethanolic } \\
\text { extract }\end{array}$ & Immunostimulant & Deshmukh et al. (2015) \\
\hline & & Methanolic extract & Immunostimulant & Sudha et al. (2010) \\
\hline & & Ethanolic extract & Immunostimulant & Gupta et al. (2010) \\
\hline & & Seed kernels & Anti-asthmatic & Agrawal and Mehta (2008) \\
\hline & & $\begin{array}{l}\text { Methanolic extract and low } \\
\text { doses of gamma radiation }\end{array}$ & $\begin{array}{l}\text { Alleviated amiodarone- } \\
\text { induced lung toxicity }\end{array}$ & Hasan et al. (2016) \\
\hline & & n-Butanol extract of seeds & $\begin{array}{l}\text { Tidal volume is decreased } \\
\text { and respiration rate is } \\
\text { increased }\end{array}$ & Mahajan et al. (2009) \\
\hline \multirow{7}{*}{$\begin{array}{l}\text { Camellia } \\
\text { sinensis or } \\
\text { Tea (Cha) }\end{array}$} & \multirow{7}{*}{$\begin{array}{l}\text { Camellia sinensis } \\
\text { (L.) Kuntze } \\
\text { (Theaceae) }\end{array}$} & Root extract & $\begin{array}{l}\text { Anti-inflammatory, } \\
\text { Immunostimulant }\end{array}$ & $\begin{array}{l}\text { Chattopadhyay et al. } \\
\text { (2004) }\end{array}$ \\
\hline & & $\begin{array}{l}\text { Two saponins ts- } 1 \& \text { ts- } 2 \\
\text { isolated from root extract }\end{array}$ & Anti-inflammatory & Sur et al. (2001) \\
\hline & & $\begin{array}{l}\text { Ethanol extract \& } \\
\text { apigallocethechin gallate }\end{array}$ & Anti-inflammatory & Novilla et al. (2017) \\
\hline & & Leaves & Antiviral & Zaher et al. (2008) \\
\hline & & Powder & Immunostimulant & Abbas et al. (2017) \\
\hline & & Leave extract & Immunostimulant & Rahayu et al. (2018) \\
\hline & & Aqueous extract & Potent anti-asthmatic & Heo et al. (2008) \\
\hline \multirow{10}{*}{$\begin{array}{l}\text { Turmeric } \\
\text { (Holud) }\end{array}$} & \multirow{10}{*}{$\begin{array}{l}\text { Curcuma longa L. } \\
\text { (Zingiberaceae) }\end{array}$} & Curcumin & Anti-inflammatory & Akram et al. (2010) \\
\hline & & Essential oil of turmeric & Anti-inflammatory & Liju et al. (2011) \\
\hline & & Aqueous extract & Antiviral & Kim et al. (2009) \\
\hline & & Alcoholic extract & Antiviral & Ichsyani et al. (2017) \\
\hline & & Curcumin & Immunostimulant & Antony et al. (1999) \\
\hline & & Turmerones & Immunostimulant & Yue et al. (2010) \\
\hline & & Aqueous extract & Immunostimulant & Sengupta et al. (2011) \\
\hline & & N-hexane extract & Activity against lung cancer & Mohammad et al. (2010) \\
\hline & & Curcumin & $\begin{array}{l}\text { Protective effect on lps- } \\
\text { induced acute lung injury }\end{array}$ & Kim et al. (2016) \\
\hline & & Curcumin and cyclocurcumin. & $\begin{array}{l}\text { Found to be potentially } \\
\text { active in-silico against sars- } \\
\text { cov-2 protease enzymes. } \\
\text { Cyclocurcumin was found to } \\
\text { be more potent in this regard } \\
\text { than remdesivir, an FDA- } \\
\text { approved (for emergency } \\
\text { use) drug for COVID-19. }\end{array}$ & Rajagopal et al. (2020) \\
\hline
\end{tabular}


anti-inflammatory effect on lungs, precisely due to the presence of catechin (Rahardiyan, 2019). Catechin is assumed to be active against SARS-CoV2 protease and with some other immune booster, prepared tea, especially green tea, can be used as an initial preventive measure against COVID-19 (Das et al., 2020).

Turmeric (Curcuma longa L., Family: Zingiberaceae): Turmeric ('Holud' in Bangali language) roots (dried and powdered) have been used as a spice, a dye, and a herbal drug for a long time. Studies have reported it's anti-inflammatory, anticancer, antioxidant, hypolipidemic, and other health benefits. In-silico studies found that several constituents of this plant, e.g., curcumin and cyclocurcumin, showed strong binding potential to the active site of the COVID-19 causing virus. Cyclocurcumin was found to be more potent in this regard than remdesivir, a FDA-approved (for emergency use) drug for COVID-19 (Rajagopal et al., 2020). Its immune-enhancing property may help the people fight the virus (Kumar and Chander, 2020).

The reported medicinal plant materials can be used for the preparation of complementary and alternative medicines or can be used individually or collectively with proper scientific evidence of Pharmacological and Toxicological studies for the prevention and treatment of COVID-19 infections.

\section{Conclusion}

From the information depicted in the Table1, it is clear that presented plants have scientific evidences to be effective against inflammation, potentiate immunity, counteract viral infection and overcome respiratory distress syndrome to an extent and hence can be used for the preparation of complementary and alternative medicaments for the prevention and treatment of those diseases. It opens the gateway for extensive research to evaluate their potentiality against corona virus, which might lead us to get some lead compounds. Besides, herbal formulators might use this information to formulate new medicaments for treating COVID-19 pathological states. These plants might improve the health conditions before and after COVID-19 infection of general peopleif used as a supplement. This review thus will educate people regarding the potentials and benefits of medicinal plants. Along with vaccines and other medicaments, it might impose the protection and boost the healing against coronavirus infection.

\section{List of Abbreviations}

COVID-19: Coronavirus disease 2019; SARSCoV-2: Severe acute respiratory syndrome coronavirus-2; MERS: Middle Eastern respiratory syndrome; RT-PCR: Reverse transcription polymerase chain reaction; CT: Computer tomography; NSPS: Non-structural proteins; RNA: Ribonucleic acid; HIV: Human immunodeficiency virus, TCM: Traditional Chinese medicine

\section{Declarations}

Ethics approval and consent to participate: Not applicable

Consent for publication: Not applicable

Availability of data and material: All data generated or analysed during this study are included in this article. However, the datasets used and/or analysed during the current study are available from the corresponding author on reasonable request.

Competing interests: The authors declare that they have no competing interests.

Funding: The authors declare that they did not receive any financial support or funds from any organization for this project and to produce this article.

\section{Authors' contributions:}

MMRS and MAR conceptualized the project. SS and MAS wrote the manuscript draft. TJ, STM and RR performed extensive literature search and collected relevant articles. MSR critically reviewed the manuscript. MMRS and MAR critically evaluated, thoroughly revised the manuscript and supervised the project. 


\section{Acknowledgments}

The authors are grateful to the Department of Pharmacy, State University of Bangladesh, for providing necessary supports to produce this article.

\section{References}

Abbas, A., Iqbal, Z., Abbas, R.Z., Khan, M.K., Khan, J.A., Hussain, K., Mahmood, M.S. and Rizwan, H.M. 2017. Immunomodulatory effects of Camellia sinensis against coccidiosis in chickens. J. Anim. Plant Sci. 27, 415-421.

Agrawal, B. and Mehta, A. 2008. Antiasthmatic activity of Moringa oleifera Lam: A clinical study. Indian J. Pharmacol. 40, 28-31.

Aimbire, F., Penna, S.C., Rodrigues, M., Rodrigues, K.C., Lopes-Martins, R.A.B. and Sertié, J.A.A. 2007. Effect of hydroalcoholic extract of Zingiber officinalis rhizomes on LPS-induced rat airway hyperreactivity and lung inflammation. Prostaglandins Leukot. Essent. Fatty Acids 77, 129-138.

Ajala, O.S., Jukov, A. and Ma, C.M. 2014. Hepatitis C virus inhibitory hydrolysable tannins from the fruits of Terminalia chebula. Fitoterapia. 99, 117-123.

Akram, M., Shahab-Uddin, A.A., Usmanghani, K., Hannan, A., Mohiuddin, E. and Asif, M. 2010. Curcuma longa and curcumin: a review article. Rom. J. Biol. Plant Biol. 55, 65-70.

Alam, S., Kamal, T.B., Sarker, M.M.R., Zhou, J.R., Rahman, S.M.A. and Mohamed, I.N. 2021a. Therapeutic Effectiveness and Safety of Repurposing Drugs for the Treatment of COVID-19: Position Standing in 2021. Front Pharmacol. 12:659577.

Alam, S., Sarker, M.M.R., Afrin, S., Richi, F.T., Zhao, C., Zhou, J.R. and Mohamed, I.N. 2021b. Traditional Herbal Medicines, Bioactive Metabolites, and Plant Products Against COVID-19: Update on Clinical Trials and Mechanism of Actions. Front. Pharmacol. 12, 671498.

Alam, S., Emon, N.U., Shahriar, S., Richi, F.T., Haque, M.R., Islam, M.N., Sakib, S.A. and Ganguly, A. 2020. Pharmacological and computer-aided studies provide new insights into Millettia peguensis Ali (Fabaceae). Saudi Pharm. J. 28, 1777-1790.

Al-Ghamdi, M.S. 2001. The anti-inflammatory, analgesic and antipyretic activity of Nigella sativa L. J. Ethnopharmacol. 76, 45-48.

Alhakmani, F., Kumar, S. and Khan, S.A. 2013. Estimation of total phenolic content, in-vitro antioxidant and antiinflammatory activity of flowers of Moringa oleifera. Asian Pac. J. Trop. Biomed. 3, 623-627.
Amri, M. and Touil-Boukoffa, C. 2016. In-vitro antihydatic and immunomodulatory effects of ginger and [6]-gingerol. Asian Pac. J. Trop. Med. 9, 749-756.

Antonisamy, P., Agastian, P., Kang, C.-W., Kim, N.S. and Kim, J.-H. 2019. Anti-inflammatory activity of rhein isolated from the flowers of Cassia fistula L. and possible underlying mechanisms. Saudi J. Biol. Sci. 261, 96-104.

Antony, S., Kuttan, R. and Kuttan, G. 1999. Immunomodulatory activity of curcumin. Immunol. Invest. 28, 291-303.

Aravind, S.R., Joseph, M.M., Varghese, S., Balaram, P. and Sreelekha, T.T. 2012. Antitumor and immuno-.

potentiating activity of polysaccharide PST001 isolated from the seed kernel of Tamarindus indica: an in-vivo study in mice. Sci. World J. 2012, 361382.

Arifan, F., Broto, R.W., Sapatra, E.F. and Pujiastuti, A.2021. Utilization of Moringa oleifera leaves for making hand sanitizers to prevent the spread of COVID-19 virus. IOP Conference Series: Earth and Environ Sci. 623, 012015.

Sultana, A., Shahriar, S., Tahsin, M.R., Mim, S.R., Fatema, K.R., Saha, A., Yesmin, F., Bahar, N.B., Samodder, M., Mamun, M.A.H., Rahman, M.A., Ferdousy, S., Akter, T., Aktar, F., Kuddus, M.R., Rahman, M.M., Sarker, M.M.R., Büyüker, S.M., Chowdhury, J.A., Chowdhury, A.A., Kabir, S., Hossain, M.A. and Amran, M.S. 2021. A Retrospective Cross-Sectional Study Assessing Self-Reported Adverse Events following Immunization (AEFI) of the COVID-19 Vaccine in Bangladesh. Vaccines (Basel). 9:1090.

Arora, M., Rahar, S., Rageeb, M. and Nagpal, N. 2016. Phytopharmacological importance of traditional healer tree: Golden Shower. Int. J. Pharm. Life Sci.75, 50515061.

Bachiega, T.F., de Sousa, J.P.B., Bastos, J.K. and Sforcin, J.M. 2012. Clove and eugenol in noncytotoxic concentrations exert immunomodulatory/antiinflammatory action on cytokine production by murine macrophages. J. Pharmacy. Pharmacol. 64, 610-616.

Bag, A., Bhattacharyya, S., Pal, N. and Chattopadhyay, R. 2013. Anti-inflammatory, anti-lipid peroxidative, antioxidant and membrane stabilizing activities of hydroalcoholic extract of Terminalia chebula fruits. Pharm. Biol. 51, 1515-1520.

Baidya, B.K. and Sethy, P. 2020. Importance of fruits and vegetables in boosting our immune system amid the COVID 19. Food Sci. Rep. 1, 50-55. 
Banerjee, S., Srivastava, S. and Giri, A.K. 2020.Possible nutritional approach to cope with COVID-19 in Indian perspective. Adv Res J Med Clin Sci. 6, 207-219.

Bassiri-Jahromi, S., 2018. Punica granatum (Pomegranate) activity in health promotion and cancer prevention. Oncol Rev. 12, 345.

Birla, H., Rai, S. N., Singh, S.S., Zahra, W., Rawat, A., Tiwari, N., Singh, R.K., Pathak, A. and Singh, S.P. 2019. Tinospora cordifolia suppresses neuroinflammation in parkinsonian mouse model. Neuromolecular Med. 211, 42-53.

Bishayi, B., Roychowdhury, S., Ghosh, S. and Sengupta, M. 2002. Hepatoprotective and immunomodulatory properties of Tinospora cordifolia in $\mathrm{CCl} 4$ intoxicated mature albino rats. J. Toxicol. Sci. 273, 139-146.

Boskabady, M.-H., Keyhanmanesh, R., Khameneh, S., Doostdar, Y. and Khakzad, M.-R. 2011. Potential immunomodulation effect of the extract of Nigella sativa on ovalbumin sensitized guinea pigs. $J$. Zhejiang Univ. Sci. B123, 201-209.

Cai, Z., Zhang, G., Tang, B., Liu, Y., Fu, X. and Zhang, X. 2015. Promising anti-influenza properties of active constituent of Withania somnifera ayurvedic herb in targeting neuraminidase of H1N1 influenza: computational study. Cell Biochem. Biophys. 723, 727-739.

Cao, H., Wen, J., Nie, Y.L. and Xu, M. 2008. Effect of Allium sativum (garlic) diallyl disulfide (DADS) on human non-small cell lung carcinoma H1299 cells.Trop. Biomed. 251, 37-45.

Carrasco, F.R., Schmidt, G., Romero, A.L., Sartoretto, J.L., Caparroz-Assef, S.M., Bersani-Amado, C.A. and Cuman, R.K.N.2009. Immunomodulatory activity of Zingiber officinale Roscoe, Salvia officinalis L. and Syzygium aromaticum L. essential oils: evidence for humor-and cell-mediated responses. J. Pharm. Pharmacol. 617, 961-967.

Chaerunisaa, A.Y., Susilawati, Y., Muhaimin, M., Milanda, T., Hendriani, R. and Subarnas, A. 2020. Antibacterial activity and subchronic toxicity of Cassia fistula L. barks in rats.Toxicol Rep.7, 649-657.

Chakotiya, A.S., Tanwar, A., Srivastava, P., Narula, A. and Sharma, R.K. 2017. Effect of aquo-alchoholic extract of Glycyrrhiza glabra against Pseudomonas aeruginosa in Mice Lung Infection Model. Biomed. Pharmacother. 90, 171-178.

Chandra, S., Chatterjee, P., Dey, P. and Bhattacharya, S. 2012. Evaluation of anti-inflammatory effect of ashwagandha: a preliminary study in-vitro. Pharmacogn. J. 429, 47-49.
Chang, J.S., Wang, K.C., Yeh, C.F., Shieh, D.E. and Chiang, L.C. 2013. Fresh ginger (Zingiber officinale) has antiviral activity against human respiratory syncytial virus in human respiratory tract cell lines. $J$. Ethnopharmacol. 145, 146-151.

Chattopadhyay, P., Besra, S.E., Gomes, A., Das, M., Sur, P., Mitra, S. and Vedasiromoni, J.R. 2004. Antiinflammatory activity of tea (Camellia sinensis) root extract. Life Sci. 74, 1839-1849.

Chavan, R. and Chowdhary, A. 2014. In-vitro inhibitory activity of Justicia adhatoda extracts against influenza virus infection and hemagglutination. Int. J. Pharm. Sci. Rev. Res. 25, 231-236.

Chen, H., Pu, J., Liu, D., Yu, W., Shao, Y., Yang, G., Xiang, Z. and He, N. 2016. Anti-inflammatory and antinociceptive properties of flavonoids from the fruits of black mulberry (Morus nigra L.). PloS One 11, $\mathrm{e} 0153080$.

Choi, H.-J. 2018. Chemical constituents of essential oils possessing anti-influenza A/WS/33 virus activity. Osong Public Health Res. Perspect 9, 348-353.

Chollom, S.C., Agada, G.O.A., Gotep, J.G., Mwankon, S.E., Dus, P.C., Bot, Y.S., Nyango, D.Y., Singnap, C.L., Fyaktu, E.J. and Okwori, A.E.J. 2012. Investigation of aqueous extract of Moringa oleifera Lam seed for antiviral activity against newcastle disease virus in ovo. J. Med. Plants Res. 6, 3870-3875.

Choudhary, P., Chakdar, H., Singh, D., Selvaraj, C., Singh, S.K., Kumar, S. and Saxena, A.K. 2020. Computational studies reveal piperine, the predominant oleoresin of black pepper (Piper nigrum) as a potential inhibitor of SARS-CoV-2 (COVID-19). Curr. Sci. 119, 1333-1342.

Chouhan, Y.S., Kataria, H.C. and Gosawmi, C.S. 2014. Anti-inflammatory activity of methanolic extract of Brassica juncea seed on carrageenan induced paw edema in rats. Int. J. Pharm. Sci. Res. 5, 3849.

Chowdhury, P. 2020. In-silico investigation of phytoconstituents from Indian medicinal herb 'Tinospora cordifolia (giloy)' against SARS-CoV-2 (COVID-19) by molecular dynamics approach. $J$. Biomol. Struct. Dyn. 7, 1-18.

Churiyah, Pongtuluran, O.B., Rofaani, E. and Tarwadi. 2015. Antiviral and immunostimulant activities of Andrographis paniculata. HAYATI J. Biosci. 22, $67-$ 72.

Coon, J.T. and Ernst, E. 2004. Andrographis paniculata in the treatment of upper respiratory tract infections: a systematic review of safety and efficacy. Planta Med. 70, 293-298. 
Das, M., Banerji, A., Cheemalapati, V.N. and Hazra, J. 2020. Antiviral Activity Of Indian Medicinal Plants: Prventive Measures For COVID-19. J Glob Biosci. 9, 7307-7319.

Dave, G.S., Rakholiya, K.D., Kaneria, M.J., Galvadiya, B.P., Vyas, S.R., Kanbi, V.H. and Patel, M.P. 2020. High affinity interaction of Solanum tuberosum and Brassica juncea residue smoke water compounds with proteins involved in coronavirus infection. Phytother. Res. 34, 3400-3410.

De, C.E., Halamová, K. and Van, D.P. 2009. Tamarind (Tamarindus indica L.): a review of traditional uses, phytochemistry and pharmacology. ACS Symposium Series, 1021, 85-110.

Deshmukh, P., Sharma, R.K., Sharma, V. and Jain, P. 2015. Immunomodulatory activity of Moringa oleifera in albino rats. J. Anim. Res. 5, 277-281.

Diwan, P.V., Margaret, I., and Ramakrishna, S. 1995. Influence of Gymnema sylvestre on inflammation. Inflammopharmacol. 33, 271-277.

Donma, M.M. and Donma, O. 2020. The effects of Allium sativum on immunity within the scope of COVID-19 infection. Med. Hypothes. 144, 109934.

El Tahir, K.E., Ashour, M.M. and Al-Harbi, M.M. 1993. The respiratory effects of the volatile oil of the black seed (Nigella sativa) in guinea-pigs: elucidation of the mechanism(s) of action. Gen. Pharmacol. Vasc. Syst. 245, 1115-1122.

Elberry, A.A., Mufti, S., Al-Maghrabi, J., Sattar, E.A., Ghareib, S.A., Mosli, H.A. and Gabr, S.A. 2014. Immunomodulatory effect of red onion (Allium cepa Linn) scale extract on experimentally induced atypical prostatic hyperplasia in Wistar rats. Mediators Inflamm. 14, 640746.

Emon, N.U., Alam, S., Rudra, S., Riya, S.R., Paul, A., Hossen, S.M., Kulsum, U. and Ganguly, A. 2021. Antidepressant, anxiolytic, antipyretic, and thrombolytic profiling of methanol extract of the aerial part of Piper nigrum: In vivo, in vitro and in silico approaches. Food Sci Nutr. 9, 833-846.

Esquenazi, D., Wigg, M.D., Miranda, M.M.F.S., Rodrigues, H.M., Tostes, J.B.F., Rozental, S., da Silva, A.J.R. and Alviano, C.S. 2002. Antimicrobial and antiviral activities of polyphenolics from Cocos nucifera Linn. (Palmae) husk fiber extract.Res. Microbiol. 153, 647-652.

Fabricant D.S. and Farnsworth N.R. 2001. The value of plants used in traditional medicine for drug discovery. Environ. Health Perspect. 109 (Suppl 1), 69-75.
Faccin-Galhardi, L.C., Aimi Yamamoto, K., Ray, S., Ray, B., Carvalho Linhares, R.E. and Nozawa, C. 2012.The in-vitro antiviral property of Azadirachta indica polysaccharides for poliovirus.J. Ethnopharmacol. 142, 86-90.

Fitriani, I.N., Utami, W., Zikri, A.T. and Santoso, P. 2020. In-silico approach of potential phytochemical inhibitor from Moringa oleifera, Cocos nucifera, Allium cepa, Psidium guajava, and Eucalyptus globulus for the treatment of COVID-19 by molecular docking (preprint). Preprint (Research Square). doi:10.21203/ rs.3.rs-42747/v1.

Frattaruolo, L., Carullo, G., Brindisi, M., Mazzotta, S., Bellissimo, L., Rago, V., Curcio, R., Dolce, V., Aiello, F. and Cappello, A.R. 2019. Antioxidant and antiinflammatory activities of flavanones from Glycyrrhiza glabra L. (licorice) leaf phytocomplexes: Identification of licoflavanone as a modulator of NFkB/MAPK pathway. Antioxidants 8, 186.

Gaber, D.A. and Badawy, W.A. 2019. Role of flaxseed oil and silymarin in amelioration of lead-induced kidney injury. Kasr Al Ainy Med. J. 25, 29-37.

Ghannadi, A., Hajhashemi, V. and Jafarabadi, H. 2005.An investigation of the analgesic and anti-inflammatory effects of Nigella sativa seed polyphenols. J. Med. Food 8, 488-493.

Ghoke, S.S., Sood, R., Kumar, N., Pateriya, A.K., Bhatia, S., Mishra, A., Dixit, R., Singh, V.K., Desai, D.N. and Kulkarni, D.D. 2018. Evaluation of antiviral activity of Ocimum sanctum and Acacia arabica leaves extracts against $\mathrm{H} 9 \mathrm{~N} 2$ virus using embryonated chicken egg model. BMC Complement. Altern. Med. 18, 1-10.

Ghosh, R., Chakraborty, A., Biswas, A. and Chowdhuri, S. 2020. Identification of alkaloids from Justicia adhatoda as potent SARS CoV-2 main protease inhibitors: An in-silico perspective. J. Mol. Struct. 1229, 129489.

Graziose R., Ann Lila M. and Raskin I. 2010.Merging traditional Chinese medicine with modern drug discovery technologies to find novel drugs and functional foods. Curr. Drug Discov. Technol. 7, 2-12.

Gupta, A., and Singh, S. 2014. Evaluation of antiinflammatory effect of Withania somnifera root on collagen-induced arthritis in rats. Pharm. Biol. 52, 308-320.

Gupta, A., Gautam, M.K., Singh, R.K., Kumar, M.V., Rao, C.V., Goel, R.K. and Anupurba, S. 2010. Immunomodulatory effect of Moringa oleifera Lam. extract on cyclophosphamide induced toxicity in mice. Ind. J. Exp. Biol. 48, 1157-1160. 
Haidari, M., Ali, M., Ward Casscells, S. and Madjid, M. 2009. Pomegranate (Punica granatum) purified polyphenol extract inhibits influenza virus and has a synergistic effect with oseltamivir. Phytomedicine 16, 1127-1136.

Haque, E., Karim, A., Chowhdury, J.A., Rezwan, R., Akter, T., Tahsin, R., Chowdhury, A., Kabir, S. and Shah, M. 2021. Can Terminalia Chebula (Haritaki) Stop COVID-19? Eur. J. Pharm. Med. Res. 8, 115-119.

Haridas, M., Sasidhar, V., Nath, P., Abhithaj, J., Sabu, A. and Rammanohar, P. 2021. Compounds of Citrus medica and Zingiber officinale for COVID-19 inhibition: in-silico evidence for cues from Ayurveda. Future J. Pharm. Sci.7, 13.

Harikrishnan, R., Balasundaram, C., Jawahar, S. and Heo, M.-S.2012. Immunomodulatory effect of Withania somnifera supplementation diet in the giant freshwater prawn Macrobrachium rosenbergii (de Man) against Aeromonas hydrophila. Fish Shellfish Immunol. 32, 94-100.

Hasan, H., Thabet, N.M. and Abdel-Rafei, M.K. 2016. Methanolic extract of Moringa oleifera leaf and low doses of gamma radiation alleviated amiodaroneinduced lung toxicity in albino rats. Arch. Biol. Sci.68, 31-39.

Heo, J.-C., Rho, J.R., Kim, T.-H., Kim, S.-Y. and Lee, S.H. 2008. An aqueous extract of green tea Camellia sinensis increases expression of Th1 cell-specific antiasthmatic markers. Int. J. Mol. Med. 22, 763-767.

Hossain, M.J. and Rahman, S.M.A. 2020. Repurposing therapeutic agents against SARS-CoV-2 infection: most promising and neoteric progress. Expert Rev. Anti. Infect. Ther. 18, 1-19.

Hossain, M.J., Kuddus, M.R. and Rahman, S.A. 2021. Knowledge, attitudes, and behavioral responses toward COVID-19 during early phase in Bangladesh: a questionnaire-based study. Asia Pac. J. Public Health 33, 141-144.

Hossain, M.J., Kuddus, M.R., Rashid, M.A. and Sultan, M.Z. 2021. Understanding and dealing the SARSCoV-2 infection: an updated concise review. Bangladesh Pharm. J. 24, 61-75.

Hu, B., Guo, H., Zhou, P. and Shi, Z. L. 2020. Characteristics of SARS-CoV-2 and COVID-19.Nat. Rev. Microbiol. 19, 141-154.

Huber, R., Stintzing, F.C., Briemle, D., Beckmann, C., Meyer, U. and Gründemann, C. 2012. In-vitro antiallergic effects of aqueous fermented preparations from citrus and cydonia fruits. Planta Med. 78, 334340.
Hussain, K., Iqbal, Z., Abbas, R.Z., Khan, M.K. and Saleemi, M.K. 2017. Immunomodulatory activity of Glycyrrhiza glabra extract against mixed Eimeria infection in chickens. Int. J. Agric. Biol. 19, 928-932.

Hussein, H.J., Hameed, I.H. and Hadi, M.Y. 2017. A Review: Anti-microbial, anti-inflammatory effect and cardiovascular effects of garlic: Allium sativum. Res. J. Pharm. Technol. 10, 4069-4078.

Ichsyani, M., Ridhanya, A., Risanti, M., Desti, H., Ceria, R., Putri, D.H., Sudiro, T.M. and Dewi, B.E. 2017.Antiviral effects of Curcuma longa L. against dengue virus in-vitro and in-vivo.in: IOP Conf. Ser.: Earth Environ. Sci. 101, 012005.

Ilavarasan, M.M. and Venkataraman, S. 2005. Antiinflammatory and antioxidant activities of Cassia fistula Linn bark extracts. Afr. J. Tradit. Complement. Altern. Med. 2, 70-85.

Inouye, S., Yamaguchi, H. and Takizawa, T. 2001. Screening of the antibacterial effects of a variety of essential oils on respiratory tract pathogens, using a modified dilution assay method. J. Infect. Chemother. 7, 251-254.

Israfil, S.M., Sarker, M.M.R., Rashid, P.T., Talukder, A.A., Kawsar, K.A., Khan, F., Akhter, S., Poh, C.L., Mohamed, I.N. and Ming, L.C. 2021. Clinical characteristics and diagnostic challenges of COVID-19: an update from the global perspective. Front Public Health 8, 955.

Jadhav, S.N. 2014.Evaluation of immunomodulatory activity of Cassia fistula.Int J Pharm Chem Biol Sci. 3, 291-293.

Jeba, C.R., Vaidyanathan, R. and Rameshkumar, G. 2011. Immunomodulatory activity of aqueous extract of Ocimum sanctum in rat.,Int. J. Pharm. Biomed. Res. 2, 33-38.

Jeena, K., Liju, V.B., Umadevi, N.P. and Kuttan, R. 2014. Antioxidant, anti-inflammatory and antinociceptive properties of black pepper essential oil (Piper nigrum Linn). J. Essent. Oil Bear. Plants 17, 1-12.

Kaisar M.A., Rahman M.S., Rahman M.Z., Hasan C.M. and Rashid M.A. 2011. A review on phytochemicals from some medicinal plants of Bangladesh. J. Phar. Nutri. Sci. 1, 87-95.

Kaithwas, G., Mukherjee, A., Chaurasia, A.K. and Majumdar, D.K. 2011. Anti-inflammatory, analgesic and antipyretic activities of Linum usitatissimum L. (flaxseed/linseed) fixed oil. Ind. J. Exp. Biol. 49, 932938. 
Kambizi, L.G., Goosen, B.M., Taylor, M.B. and Afolayan, A.J. 2007. Anti-viral effects of aqueous extracts of Aloe ferox and Withania somnifera on herpes simplex virus type lin cell culture. S. Afr. J. Sci. 103, 359-360.

Kanyinda, J.N. 2020. Coronavirus (COVID-19): a protocol for prevention and treatment (Covalyse $\left.{ }^{\circledR}\right)$. Eur. $J$. Med. Health. Sci. 2, 1-4.

Karetha, K., Gadhvi, K., and Vyas, S.2020. Peelings of citrus fruits as a precious resource of phytochemical and vital bioactive medicines during Covid: 19 periods.Int. J. Bot. Stud. 5, 342-344.

Karimi, M., Sadeghi, R. and Kokini, J. 2017.Pomegranate as a promising opportunity in medicine and nanotechnology. Trends Food Sci. Technol. 69, 59-73.

Kasote, D.M., Zanwar, A.A., Devkar, S.T., Hegde, M.V. and Deshmukh, K.K. 2012.Immunomodulatory activity of ether insoluble phenolic components of nbutanol fraction (EPC-BF) of flaxseed in rat.Asian Pac. J. Trop. Biomed. 2, S623-S626.

Kaur, G., Singh, N., Samuel, S.S., Bora, H.K., Sharma, S., Pachauri, S.D., Dwivedi, A.K., Siddiqui, H.H. and Hanif, K. 2015. Withania somnifera shows a protective effect in monocrotaline-induced pulmonary hypertension. Pharm. Biol. 53, 147-157.

Kaur, R., Ruhil, S., Balhara, M., Dhankhar, S. and Chhillar, A.K. 2013. A review on Justicia adhatoda: A potential source of natural medicine. Afr. J. Plant Sci. 5, 620-627.

Kaushik, Sulochana, Jangra, G., Kundu, V., Yadav, J.P. and Kaushik, Samander 2020. Antiviral activity of Zingiber officinale (Ginger) ingredients against the Chikungunya virus.Virus disease 31, 270-276.

Kesharwani, A., Polachira, S.K., Nair, R., Agarwal, A., Mishra, N.N. and Gupta, S.K. 2017. Anti-HSV-2 activity of Terminalia chebula Retz extract and its constituents, chebulagic and chebulinic acids. BMC Complement. Altern. Med. 17, 1-11.

Keyaerts, E., Vijgen, L., Pannecouque, C., Van Damme, E., Peumans, W., Egberink, H., Balzarini, J. and Van Ranst, M. 2007. Plant lectins are potent inhibitors of coronaviruses by interfering with two targets in the viral replication cycle. Antiviral Res.75, 179-187.

Khan, F., Sarker, M., Rahman, M., Ming, L.C., Mohamed, I.N., Zhao, C., Sheikh, B.Y., Tsong, H.F., and Rashid, M.A. 2019.Comprehensive review on phytochemicals, pharmacological and clinical potentials of Gymnema sylvestre. Front. Pharmacol. 10, 1223.

Khanna, K., Kohli, S. K., Kaur, R., Bhardwaj, A., Bhardwaj, V., Ohri, P., Sharma, A., Ahmad, A., Bhardwaj, R. and Ahmad, P. 2021. Herbal immuneboosters: Substantial warriors of pandemic Covid-19 battle. Phytomed. 85: 153361.
Khanna, V.G., Kannabiran, K., Babu, V.S. and Hameed, A.S. 2011. Inhibition of fish nodavirus by gymnemagenol extracted from Gymnema sylvestre. J. Ocean Univ. China 10, 402-408.

Khubber, S., Hashemifesharaki, R., Mohammadi, M. and Gharibzahedi, S.M.T. 2020. Garlic (Allium sativum L.): a potential unique therapeutic food rich in organosulfur and flavonoid compounds to fight with COVID-19. Nutr. J. 19, 124.

Killedar, S.G. and Nale, A.B., 2014. Isolation, characterization, and evaluation of Cassia fistula Linn.seed and pulp polymer for pharmaceutical application. Int. J. Pharm. Invest. 4, 215-225.

Kim, H.J., Yoo, H.S., Kim, J.C., Park, C.S., Choi, M.S., Kim, M., Choi, H., Min, J.S., Kim, Y.S. and Yoon, S.W. 2009. Antiviral effect of Curcuma longa Linn extract against hepatitis B virus replication. $J$. Ethnopharmacol. 124, 189-196.

Kim, J., Jeong, S.-W., Quan, H., Jeong, C.-W., Choi, J.-I. and Bae, H.-B. 2016. Effect of curcumin (Curcuma longa extract) on LPS-induced acute lung injury is mediated by the activation of AMPK. J. Anesth. 30, 100-108.

Kim, S.-H. and Lee, Y.-C. 2009. Piperine inhibits eosinophil infiltration and airway hyperresponsiveness by suppressing $\mathrm{T}$ cell activity and Th2 cytokine production in the ovalbumin-induced asthma model. $J$. Pharm. Pharmacol. 61, 353-359.

Koshak, D.A.E. and Koshak, P.E.A. 2020. Nigella sativa L as a potential phytotherapy for coronavirus disease 2019: A mini review of in-silico studies. Curr.Ther.Res. 93, 100602.

Kumar, G. and Chander, H. 2020. Scope for Utilization of Curcuma longa, Emblica officinalis and Tinospora cordifolia for Human Health in COVID-19 Like Scenario in Himachal Pradesh. India. Epidemiology Transmission Infec. Dis. 1, 199-210.

Kurokawa, M., Hozumi, T., Basnet, P., Nakano, M., Kadota, S., Namba, T., Kawana, T. and Shiraki, K. 1998.Purification and characterization of eugeniin as an anti-herpesvirus compound from Geum japonicum and Syzygium aromaticum.J. Pharmacol. Exp. Ther. 284, 728-735.

Kwak, Y., Lee, J. and Ju, J. 2016. Anticancer activities of Brassica juncea leavesin-vitro. EXCLI. J. 15, 699-710.

Labsi, M., Khelifi, L., Mezioug, D., Soufli, I., and TouilBoukoffa, C. 2016. Antihydatic and immunomodulatory effects of Punica granatum peel aqueous extract in a murine model of echinococcosis. Asian Pac. J. Trop. Med. 9, 211-220. 
Latha, D., Prabu, P., Arulvasu, C., Manikandan, R., Sampurnam, S. and Narayanan, V. 2018. Enhanced cytotoxic effect on human lung carcinoma cell line (A549) by gold nanoparticles synthesized from Justicia adhatoda leaf extract. Asian Pac. J. Trop. Biomed. 8, 540-547.

Laxmi, V., Wahi, N., Goel, A. and Bhatia, A.K. 2015. Investigating the immunomodulatory effect of Cassia fistula on Albino rats. Adv. Pharm. Ethnomed. 3, 1-5.

Lee, J.C., Krochak, R., Blouin, A., Kanterakis, S., Chatterjee, S., Arguiri, E., Vachani, A., Solomides, C.C., Cengel, K.A. and Christofidou-Solomidou, M. 2009. Dietary flaxseed prevents radiation-induced oxidative lung damage, inflammation and fibrosis in a mouse model of thoracic radiation injury. Cancer Biol. Ther. 8, 47-53.

Lee, J.-W., Ryu, H.W., Park, S.-Y., Park, H., Kwon, O.K., Yuk, H.J., Shrestha, K., Park, M., Kim, J., Lee, S., Oh, S.-R. and Ahn, K.-S. 2017. Protective effects of neem (Azadirachta indica A. Juss.) leaf extract against cigarette smoke- and lipopolysaccharide-induced pulmonary inflammation. Int. J. Mol. Med. 40, 19321940.

Lee, N.-K., Lee, J.-H., Lim, S.-M., Lee, K.A., Kim, Y.B., Chang, P.-S. and Paik, H.-D. 2014. Antiviral activity of subcritical water extract of Brassica juncea against influenza virus A/H1N1 in nonfat milk. J. Dairy Sci. 97, 5383-5386.

Lee, S.A., Lee, S.H., Kim, J.Y. and Lee, W.S. 2019.Effects of glycyrrhizin on lipopolysaccharide-induced acute lung injury in a mouse model. J. Thorac. Dis. 11, 1287-1302.

Leyon, P.V., and Kuttan, G. 2004. Effect of Withania somnifera on B16F-10 melanoma induced metastasis in mice. Phytother. Res. 18, 118-122.

Li, Y., Yang, F., Zheng, W., Hu, M., Wang, J., Ma, S., Deng, Y., Luo, Y., Ye, T. and Yin, W. 2016. Punica granatum (pomegranate) leaves extract induces apoptosis through mitochondrial intrinsic pathway and inhibits migration and invasion in non-small cell lung cancer in-vitro. Biomed.Pharmacother. 80, 227-235.

Liju, V.B., Jeena, K. and Kuttan, R. 2011. An evaluation of antioxidant, anti-inflammatory, and antinociceptive activities of essential oil from Curcuma longa. L. Indian J. Pharmacol. 43, 526-531.

Lim, S.H. and Choi, C.I. 2019. Pharmacological properties of Morus nigra L. (black mulberry) as a promising nutraceutical resource. Nutrients 11, 437.

Londhe, V.P. 2011. Role of garlic (Allium sativum) in various diseases: An overview. J. Pharm. Res. Opinion 4, 129-134.
Ma, H., Diao, Y., Zhao, D., Li, K. and Kang, T. 2010. A new alternative to treat swine influenza A virus infection: extracts from Terminalia chebula Retz. African J. Micro. Res. 4, 497-499.

Magzoub, M. 2020. Life Style Guideline of Ginger (Zingiber officinale) as Prophylaxis and Treatment for Coronaviruses (SARS-CoV-2) Infection (COVID-19). Saudi J. Biomed. Res. 5, 125-127.

Mahajan, S.G., Banerjee, A., Chauhan, B.F., Padh, H., Nivsarkar, M. and Mehta, A.A. 2009. Inhibitory effect of n-butanol fraction of Moringa oleifera Lam. seeds on ovalbumin-induced airway inflammation in a guinea pig model of asthma. Int. J. Toxicol. 28, 519527.

Mahluji, S., Ostadrahimi, A., Mobasseri, M., Attari, V.E. and Payahoo, L. 2013. Anti-inflammatory effects of Zingiber officinale in type 2 diabetic patients. $A d v$. Pharm. Bull. 3, 273-276.

Mair, C.E., Liu, R., Atanasov, A.G., Schmidtke, M., Dirsch, V.M. and Rollinger, J.M. 2016.Antiviral and anti-proliferative in-vitro activities of piperamides from black pepper.Planta Med. 82, S1-S381.

Mao, Q.Q., Xu, X.Y., Cao, S.Y., Gan, R.Y., Corke, H. and Li, H.B. 2019. Bioactive compounds and bioactivities of ginger (Zingiber officinale Roscoe). Foods. 8, 185.

Mariselvam, R., Ranjitsingh, A.J.A., Usha Raja Nanthini, A., Kalirajan, K., Padmalatha, C. and Mosae Selvakumar, P. 2014. Green synthesis of silver nanoparticles from the extract of the inflorescence of Cocos nucifera (Family: Arecaceae) for enhanced antibacterial activity. Spectrochim.Acta. A. Mol. Biomol. Spectrosc. 129, 537-541.

Mascarello, A., Orbem Menegatti, A.C., Calcaterra, A., Martins, P.G.A., Chiaradia-Delatorre, L.D., D’Acquarica, I., Ferrari, F., Pau, V., Sanna, A., De Logu, A., Botta, M., Botta, B., Terenzi, H. and Mori, M. 2018. Naturally occurring Diels-Alder-type adducts from Morus nigra as potent inhibitors of Mycobacterium tuberculosis protein tyrosine phosphatase B. Eur. J. Med. Chem. 144, 277-288.

Mazumder, J.A., Khan, E., Perwez, M., Gupta, M., Kumar, S., Raza, K. and Sardar, M. 2020.Exposure of biosynthesized nanoscale $\mathrm{ZnO}$ to Brassica juncea crop plant: Morphological, biochemical and molecular aspects. Sci Rep. 10, 1-3.

Mazumder, P.M., Pattnayak, S., Parvani, H., Sasmal, D. and Rathinavelusamy, P. 2012. Evaluation of immunomodulatory activity of Glycyrhiza glabra L roots in combination with zing. Asian Pac. J. Trop. Biomed.2, S15-S20. 
Mediratta, P.K., Sharma, K.K. and Singh, S. 2002. Evaluation of immunomodulatory potential of Ocimum sanctum seed oil and its possible mechanism of action. J. Ethnopharmacol. 80, 15-20.

Mehdi, H., Zeinab, B., Saeide, S. and Yasub, S. 2014. Antimicrobial Activity of Trachyspermum ammi Essential Oil Against Human Bacterial. Int. J. Adv. Biol. Biomed. Res.2, 18-24.

Mehmood, Y., Farooq, U., Youusaf, H., Riaz, H., Mahmood, R.K., Nawaz, A., Abid, Z., Gondal, M., Barkat, K. and Khalid, I. 2020. Antiviral activity of green silver nanoparticles produced using aqueous buds extract of Syzygium aromaticum. Pak. J. Pharm. Sci. 33, 839-845.

Meléndez-Villanueva, M.A., Morán-Santibañez, K., Martínez-Sanmiguel, J.J., Rangel-López, R., GarzaNavarro, M.A., Rodríguez-Padilla, C., Zarate-Triviño, D.G. and Trejo-Ávila, L.M. 2019.Virucidal activity of gold nanoparticles synthesized by green chemistry using garlic extract.Viruses 11, 1111.

Menegazzi, M., Di Paola, R., Mazzon, E., Genovese, T., Crisafulli, C., Dal Bosco, M., Zou, Z., Suzuki, H. and Cuzzocrea, S. 2008. Glycyrrhizin attenuates the development of carrageenan-induced lung injury in mice. Pharmacol. Res. 58, 22-31.

Menichini, F., Loizzo, M.R., Bonesi, M., Conforti, F., De Luca, D., Statti, G.A., de Cindio, B., Menichini, Francesco and Tundis, R. 2011. Phytochemical profile, antioxidant, anti-inflammatory and hypoglycemic potential of hydroalcoholic extracts from Citrus medica L. cv Diamante flowers, leaves and fruits at two maturity stages. Food Chem. Toxicol. 49, 1549-1555.

Mittal, J., Pal, U., Sharma, L., Verma, A.K., Ghosh, M. and Sharma, M.M. 2020. Unveiling the cytotoxicity of phytosynthesised silver nanoparticles using Tinospora cordifolia leaves against human lung adenocarcinoma A549 cell line. IET Nanobiotechnol. 14, 230-238.

Mittal, J., Sharma, M.M. and Batra, A. 2014. Tinospora cordifolia: a multipurpose medicinal plant-A. J. Med. Plants 2, 32-47.

Mofed, D., Ahmed, W., Zekri, A.-R., Said, O., Rahouma, M. and Faraag, A.H.I. 2020. The antiviral efficacy of Withania somnifera (Ashwagandha) against Hepatitis $\mathrm{C}$ virus activity: in-vitro and in-silico study. $A d v$. Microbiol. 10, 463-477.

Mohammad, P., Nosratollah, Z., Mohammad, R., Abbas, A. and Javad, R. 2010. The inhibitory effect of Curcuma longa extract on telomerase activity in A549 lung cancer cell line. Afr. J. Biotechnol. 9, 912-919.
Mouhajir, F., Hudson, J.B., Rejdali, M. and Towers, G.H.N. 2001.Multiple antiviral activities of endemic medicinal plants used by Berber peoples of Morocco.Pharm. Biol. 39, 364-374.

Mulla, W.A., More, S.D., Jamge, S.B., Pawar, A.M., Kazi, M.S. and Varde, M.R. 2010. Evaluation of antiinflammatory and analgesic activities of ethanolic extract of roots Adhatoda vasica Linn. Int. J. Pharm. Tech. Res. 2, 1364-1368.

Murugan, N.A., Pandian, C.J. and Jeyakanthan, J. 2020. Computational investigation on Andrographis paniculata phytochemicals to evaluate their potency against SARS-CoV-2 in comparison to known antiviral compounds in drug trials. J. Biomol. Struct.Dyn. 16, 1-12.

Nasr-Eldin, M.A., Abdelhamid, A. and Baraka, D. 2017. Antibiofilm and antiviral potential of leaf extracts from Moringa oleifera and rosemary (Rosmarinus officinalis Lam.). Egypt. J. Microbiol.52, 129-139.

Ndiaye, M., Dieye, A.M., Mariko, F., Tall, A. and Faye, B. 2002. Contribution to the study of the antiinflammatory activity of Moringa oleifera (Moringaceae).Dakar Med. 47, 210-212.

Nikhat, S. and Fazil, M. 2020. Overview of Covid-19; its prevention and management in the light of Unani medicine.Sci. Total Environ. 728, 138859.

Nirmala, P. and Selvaraj, T. 2011. Anti-inflammatory and anti-bacterial activities of Glycyrrhiza glabra L. J. Agric. Technol. 7, 815-823.

Nomura, T. 1988. Phenolic compounds of the mulberry tree and related plants. Fortschr.Chem. Org. Naturst. $\mathbf{5 3}, 87-201$.

Nosalova, G., Jurecek, L., Chatterjee, U.R., Majee, S.K., Nosal, S. and Ray, B. 2013. Antitussive activity of the water-extracted carbohydrate polymer from Terminalia chebula on citric acid-induced cough. Evid.-Based Complement. Altern. Med. 2013, 650134.

Novilla, A., Djamhuri, D.S., Nurhayati, B., Rihibiha, D.D., Afifah, E. and Widowati, W. 2017. Anti-inflammatory properties of oolong tea (Camellia sinensis) ethanol extract and epigallocatechin gallate in LPS-induced RAW 264.7 cells. Asian Pac. J. Trop. Biomed. 7, 1005-1009.

Ogadinma, I., Chuemere, A.N. and Vitalis, V. 2018. Evaluation of Aromatherapeutic Potential of Allium cepa in Carbon Monoxide-induced Respiratory Tissue Toxicity in Wistar Rats.Asian J. Res. Med. Pharm. Sci. 4, 1-7. 
Okoh, O.O., Obiiyeke, G.E., Nwodo, U.U. and Okoh, A.I. 2017. Ethanol extract and chromatographic fractions of Tamarindus indica stem bark inhibits Newcastle disease virus replication. Pharm. Biol. 55, 1806-1808.

Okpanyi, S.N. and Ezeukwu, G.C. 1981. Antiinflammatory and antipyretic activities of Azadirachta indica.Planta Med. 41, 34-39.

Oliveira, T.T., Campos, K.M., Cerqueira-Lima, A.T., Cana Brasil Carneiro, T., da Silva Velozo, E., Ribeiro Melo, I.C.A., Figueiredo, E.A., de Jesus Oliveira, E., de Vasconcelos, D.F.S.A., Pontes-de-Carvalho, L.C., Alcântara-Neves, N.M. and Figueiredo, C.A. 2015. Potential therapeutic effect of Allium cepa L. and quercetin in a murine model of Blomia tropicalis induced asthma. DARU J. Pharm. Sci. 23, 1-12.

Pandey, P., Khan, F., Kumar, A., Srivastava, A. and Jha, N.K. 2021. Screening of potent inhibitors against 2019 novel coronavirus (Covid-19) from Allium sativum and Allium cepa: an in-silico approach. Biointerface Res. Appl. Chem. 11, 7981-7993.

Pant, M., Ambwani, T. and Umapathi, V. 2012. Antiviral activity of Ashwagandha extract on infectious bursal disease virus replication. Indian J. Sci. Technol. 5, 2750-2751.

Parikh, M., Netticadan, T. and Pierce, G.N. 2018. Flaxseed: its bioactive components and their cardiovascular benefits. Am. J. Physiol. Heart Circ. 314, 146-59.

Pastorino, G., Cornara, L., Soares, S., Rodrigues, F. and Oliveira, M.B.P.P. 2018. Liquorice (Glycyrrhiza glabra): A phytochemical and pharmacological review. Phytother.Res. 32, 2323-2339.

Patgiri, B., Umretia, B.L., Vaishnav, P.U., Prajapati, P.K., Shukla, V.J. and Ravishankar, B. 2014.Antiinflammatory activity of Guduchi Ghana (aqueous extract of Tinospora Cordifolia Miers.). Ayu 35, 108110.

Pei, H., Xue, L., Tang, M., Tang, H., Kuang, S., Wang, L., Ma, X., Cai, X., Li, Y. and Zhao, M. 2020. Alkaloids from black pepper (piper nigrum L.) exhibit antiinflammatory activity in murine macrophages by

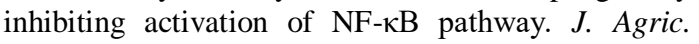
Food Chem. 68, 2406-2417.

Penna, S.C., Medeiros, M.V., Aimbire, F.S.C., Faria-Neto, H.C.C., Sertie, J.A.A. and Lopes-Martins, R.A.B. 2003. Anti-inflammatory effect of the hydralcoholic extract of Zingiber officinale rhizomes on rat paw and skin edema. Phytomed. 10, 381-385.

Pinheiro, A.J.M.C.R., Gonçalves, J.S., Dourado, Á.W.A., de Sousa, E.M., Brito, N.M., Silva, L.K., Batista, M.C.A., de Sá, J.C., Monteiro, C.R.A.V., Fernandes, E.S. and Monteiro-Neto, V.2018. Punica granatum L. leaf extract attenuates lung inflammation in mice with acute lung injury. J. Immun. Res. 2018, 6879183.
Polak, S.B., Van Gool, I.C., Cohen, D., von der Thüsen, J. H. and van Paassen, J. 2020. A systematic review of pathological findings in COVID-19: a pathophysiological timeline and possible mechanisms of disease progression. Mod.Pathol. 33, 2128-2138.

Porchezhian, E. and Dobriyal, R.M. 2003. An overview on the advances of Gymnema sylvestre: chemistry, pharmacology and patents. Die Pharmazie 58, 5-12.

Rahardiyan, D. 2019. Antibacterial potential of catechin of tea (Camellia sinensis) and its applications.Food Res. 3, 1-6.

Rahayu, R.P., Prasetyo, R.A., Purwanto, D.A., Kresnoadi, U., Iskandar, R.P. and Rubianto, M. 2018. The immunomodulatory effect of green tea (Camellia sinensis) leaves extract on immunocompromised Wistar rats infected by Candida albicans. Vet. World 11, 765-770.

Rahman M.S., Rahman M.Z., Begum B., Chowdhury R., Islam S.N. and Rashid M.A. 2011.Antidiabetic principle from Eclipta prostrata.Latin Am. J. Pharm. 30, 1656-1660.

Rahman, M.T. 2020. Potential benefits of combination of Nigella sativa and $\mathrm{Zn}$ supplements to treat COVID-19. J. Herb. Med. 23, 100382.

Rahmani, A.H. 2015. Cassia fistula Linn: Potential candidate in the health management. Pharmacog Res. 7, 217-224.

Rajagopal, K., Varakumar, P. and Baliwada, A., Byran, G. 2020. Activity of phytochemical constituents of Curcuma longa (turmeric) and Andrographis paniculata against coronavirus (COVID-19): an insilico approach. Future J. Pharm. Sci. 6, 1-10.

Rao, K.V., Gopalakrishnan, V., Loganathan, V. and Nathan, S.S. 1999. Anti-inflammatory activity of Moringa oliefera. Lam. Anc. Sci. Life 18, 195-198.

Rashid M.A., Gustafson K.R. and Boyd M.R. 2000. HIVinhibitory cembrane derivatives from a Philippines collection of the soft coral Lobophytum species.J. Nat. Prod. 63, 531-533.

Reshma, A.K. and Brindha, P. 2014. In-vitro antiinflammatory, antioxidant and nephroprotective studies on leaves of Aegle marmelos and Ocimum sanctum.Asian J. Pharm. Clin. Res.7, 121-129.

Ricci, D., Giamperi, L., Bucchini, A. and Fraternale, D. 2006. Antioxidant activity of Punica granatum fruits. Fitoterapia.77, 310-312.

Rinaldi, S., Silva, D.O., Bello, F., Alviano, C.S., Alviano, D.S., Matheus, M.E. and Fernandes, P.D. 2009. Characterization of the antinociceptive and antiinflammatory activities from Cocos nucifera L. (Palmae). J. Ethnopharmacol. 122, 541-546. 
Romeilah, R., Fayed, S. and Mahmoud, G. 2010. Chemical compositions, antiviral and antioxidant activities of seven essential oils. J. Appl. Sci. Res. 6, 50-62.

Rouhi, S.Z.T., Sarker, M.M.R., Rahmat, A., Alkahtani, S.A. and Othman, F. 2017. The effect of pomegranate fresh juice versus pomegranate seed powder on metabolic indices, lipid profile, inflammatory biomarkers, and the histopathology of pancreatic Islets of Langerhans in streptozotocin-nicotinamide induced type 2 diabetic Sprague-Dawley rats. $B M C$ Complement. Altern. Med. 17, 1-13.

Roy, S., Chaturvedi, P. and Chowdhary, A. 2015.Evaluation of antiviral activity of essential oil of Trachyspermum Ammi against Japanese encephalitis virus. Pharmacogn. Res.7, 263-267.

Sah, A.N., Juyal, V. and Melkani, A.B. 2011. Antimicrobial activity of six different parts of the plant Citrus medica Linn. Pharmacogn. J. 3, 80-83.

Saini, A., Sharma, S. and Chhibber, S. 2009. Induction of resistance to respiratory tract infection with Klebsiella pneumoniae in mice fed on a diet supplemented with tulsi (Ocimum sanctum) and clove (Syzgium aromaticum) oils. J. Microbiol. Immunol. Infect. 42, 107-113.

Samal, K.C., Praveena, J. and Behera, L. 2020. Flaxseed: The Miracle Food to Fight against COVID-19. AGROFORESTRY 4, 67.

Saneja, A., Sharma, C., Aneja, K.R. and Pahwa, R. 2010.Gymnema sylvestre (Gurmar): a review. Der. Pharm. Lett .2, 275-284.

Sarker, M.M.R. 2021. Evaluation of Red and Green Colored Bell peppers for the Production of Polyclonal IgM and IgG Antibodies in Murine Spleen Cells. Bangladesh Pharm. J. 24, 45-53.

Sarker, M. M. R. and Gohda, E. 2013. Promotion of antikeyhole limpet hemocyanin $\operatorname{IgM}$ and $\operatorname{IgG}$ antibody productions in-vitro by red bell pepper extract. $J$. Funct. Foods 5, 1918-1926.

Sarker, M.M.R. 2012.Induction of humoral immunity through the enhancement of IgM production in murine splenic cells by ethanolic extract of seed of Piper nigrum L. J.Sci. Res. 4, 65-69.

Sarker, M.M.R., Mazumder, M.E.H. and Rashid, M.H. 2011. In-vitro enhancement of polyclonal $\operatorname{IgM}$ production by ethanolic extract of Nigella sativa L. seeds in whole spleen cells of female BALB/c mice. Bangladesh Pharm. J. 14, 73-77.
Sarker, M.M.R., Ming, L.C., Sarker, M.Z.I. and Choudhuri, M.S.K. 2016.Immunopotentiality of Ayurvedic polyherbal formulations "Saribadi" and "Anantamul Salsa" with augmentation of IgM production and lymphocytes proliferation: A preliminary study. Asian Pac. J. Trop. Biomed. 6, 568-573.

Sarker, M.M.R., Zihad, M.A.T.R., Islam, M., Nahar, M., Islam, M.M., Imam, H., Ghosh, A., Mustapha, M.S. and Ismail, N.E. 2015.Antihyperglycemic, insulinsensitivity and anti-hyperlipidemic potential of Ganoderma lucidum, a dietary mushroom, on alloxanand glucocorticoid-induced diabetic Long-Evans rats. Funct. Foods Health Dis. 5, 450-466.

Sengupta, M., Sharma, G.D. and Chakraborty, B. 2011. Hepatoprotective and immunomodulatory properties of aqueous extract of Curcuma longa in carbon tetra chloride intoxicated Swiss albino mice. Asian Pac. J. Trop. Biomed. 1, 193-199.

Shahriar, S. and Koly, F.J. 2021.A cross-sectional study on Bangladeshi students regarding physiological challenges of online education. Pharm. Educ. 21, 267275.

Shahriar, S., Rana, M.S., Hossain, M.S., Karim, A., Mredula, T.N., Nourin, N., Uddin, M.S. and Amran, M.S. 2021. COVID-19: epidemiology, pathology, diagnosis, treatment, and impact. Curr. Pharm. Des. 27, 1-24.

Shaji, D. 2020. Computational Identification of drug lead compounds for COVID-19 from Moringa oleifera. [Preprint]. https://doi.org/10.26434/chemrxiv. 12535913.v1.

Sheeja, K., Shihab, P.K. and Kuttan, G. 2006. Antioxidant and anti-inflammatory activities of the plant Andrographis paniculata Nees. Immunopharmacol. Immunotoxicol. 28, 129-140.

Shivaprasad, H.N., Kharya, M.D., Rana, A.C. and Mohan, S. 2006. Preliminary immunomodulatory activities of the aqueous extract of Terminalia chebula. Pharm. Biol. 44, 32-34.

Shojai, T.M., Langeroudi, A.G., Karimi, V., Barin, A. and Sadri, N. 2016. The effect of Allium sativum (Garlic) extract on infectious bronchitis virus in specific pathogen free embryonic egg. Avicenna $J$. Phytomedicine 6, 458-467.

Shree, P., Mishra, P., Selvaraj, C., Singh, S.K., Chaube, R., Garg, N. and Tripathi, Y.B. 2020. Targeting COVID19 (SARS-CoV-2) main protease through active phytochemicals of ayurvedic medicinal plants Withania somnifera (Ashwagandha), Tinospora cordifolia (Giloy) and Ocimum sanctum (Tulsi) - a molecular docking study. J. Biomol. Struct. Dyn. 27, $1-14$. 
Shruthi, R., Venkatesh, Y. and Gudipati, M. 2017. Structural and functional characterization of a novel immunomodulatory glycoprotein isolated from ajowan (Trachyspermum ammi L.). Glycoconj. J. 34, 499-514.

Siddhuraju, P. 2007. Antioxidant activity of polyphenolic compounds extracted from defatted raw and dry heated Tamarindus indica seed coat. LWT-Food Sci Tech. 40, 982-990.

Siddiqui, M.J., Aslam, A. and Khan, T. 2019. Comparison and evaluation of different seed extracts of Trachyspermum ammi for immunomodulatory effect on cell-mediated immunity through delayed-type hypersensitivity assay skin thickness method. $J$. Pharm. Bioallied Sci. 11, 43-48.

Sindhoor, K.L., Kumar, G.S., Nagarjuna, S. and Reddy, Y.P. 2012. Comparative study of anti-inflammatory activity of petroleum ether and ethanolic extracts of Brassica juncea.Int. J. Pharmatech Res. 4, 1172-1176.

Singh, R.P., Chidambara, Murthy, K.N. and Jayaprakasha, G.K. 2002. Studies on the antioxidant activity of pomegranate (Punica granatum) peel and seed extracts using in vitro models. J. Agri. Food Chem. 50, 81-86.

Singh, N., Singh, S.P., Nath, R., Singh, D.R., Gupta, M.L., Kohli, R.P. and Bhargava, K.P. 1986. Prevention of urethane-induced lung adenomas by Withania somnifera (L.)Dunal in albino mice. Int. J. Crude Drug Res. 24, 90-100.

Singh, S. 1998. Comparative evaluation of antiinflammatory potential of fixed oil of different species of Ocimum and its possible mechanism of action. Indian J. Exp. Biol. 36, 1028-1031.

Singh, V.K., Dwivedi, P., Chaudhary, B.R. and Singh, R. 2015. Immunomodulatory effect of Gymnema sylvestre (R. Br.) leaf extract: an in-vitro study in rat model. PloS One 10, e0139631.

Sinha, S.K., Prasad, S.K., Islam, M.A., Gurav, S.S., Patil, R.B., AlFaris, N.A., Aldayel, T.S., AlKehayez, N.M., Wabaidur, S.M. and Shakya, A. 2020. Identification of bioactive compounds from Glycyrrhiza glabra as possible inhibitor of SARS-CoV-2 spike glycoprotein and non-structural protein-15: a pharmacoinformatics study. J. Biomol. Struct. Dyn. 2020, 1-15.

Sreelekha, T.T., Vijayakumar, T., Ankanthil, R., Vijayan, K.K. and Nair, M.K. 1993. Immunomodulatory effects of a polysaccharide from Tamarindus indica. Anticancer Drugs. 4, 209-212.

Srivastava, A., Siddiqui, S., Ahmad, R., Mehrotra, S., Ahmad, B. and Srivastava, A.N. 2020.Exploring nature's bounty: identification of Withania somnifera as a promising source of therapeutic agents against COVID-19 by virtual screening and in-silico evaluation.J. Biomol. Struct. Dyn. 2020, 1-51.
Subramani, S.K., Gupta, Y., Manish, M. and Prasad, G.B. 2020.Gymnema Sylvestre A-Potential Inhibitor of COVID-19 Main Protease by MD Simulation Study.ChemRxiv.Preprint.doi.org/10.26434/chemrxiv. 12333251.v1.

Sudha, P., Asdaq, S.M., Dhamingi, S.S. and Chandrakala, G.K. 2010. Immunomodulatory activity of methanolic leaf extract of Moringa oleifera in animals. Indian J. Physiol. Pharmacol. 54, 133-140.

Sudhakaran, D.S., Srirekha, P., Devasree, L.D., Premsingh, S. and Michael, R.D. 2006. Immunostimulatory effect of Tinospora cordifolia Miers leaf extract in Oreochromis mossambicus.Ind. J. Exp. Biol. 44, 726732.

Sukardiman, M.E., Fadhil Pratama, M.R., Poerwono, H. and Siswodihardjo, S. 2020. The coronavirus disease 2019 main protease inhibitor from Andrographis paniculata (Burm. f) Ness.J. Adv. Pharm. Technol. Res. 11, 157-162.

Sunila, E.S. and Kuttan, G. 2004. Immunomodulatory and antitumor activity of Piper longum Linn.and piperine. J. Ethnopharmacol. 90, 339-346.

Sur, P., Chaudhuri, T., Vedasiromoni, J.R., Gomes, A. and Ganguly, D.K. 2001. Anti-inflammatory and antioxidant property of saponins of tea [Camellia sinensis (L) O. Kuntze] root extract. Phytother. Res. 15, 174-176.

Suralkar, A.A., Rodge, K.N., Kamble, R.D. and Maske, K.S. 2012.Evaluation of anti-inflammatory and analgesic activities of Tamarindus indica seeds.Int. J. Pharm. Sci. Drug Res. 4, 213-217.

Takahashi, M. and Shibamoto, T. 2008. Chemical compositions and antioxidant/anti-inflammatory activities of steam distillate from freeze-dried onion (Allium cepa L.) sprout. J. Agri. Food Chem. 56, 10462-10467.

Tanko, Y., Mohammed, A., Okasha, M.A., Umar, A.H. and Magaji, R.A. 2008. Anti-nociceptive and antiinflammatory activities of ethanol extract of Syzygium aromaticum flower bud in Wistar rats and mice. Afr. $J$. Tradit. Complement. Altern. Med. 5, 209-212.

Tayade, P.M., Ghaisas, M.M., Jagtap, S.A., and Dongre, S.H. 2009.Anti-asthmatic activity of methanolic extract of leaves of Tamarindus Indica Linn. J. Pharm. Res. 2, 944-947.

Tiwari, P.A., Pandey, I., Zamboni, P., Gemmati, D., Kanase, A., Singh, A.V. and Singh, M.P., 2020. Traditional Herbal Remedies with a Multifunctional Therapeutic Approach as an Implication in COVID-19 Associated Co-Infections. Coatings.10, 761.

Tiwari, M., Dwivedi, U.N. and Kakkar, P. 2014. Tinospora cordifolia extract modulates COX-2, iNOS, ICAM-1, pro-inflammatory cytokines and redox status in murine model of asthma. J. Ethnopharmacol.153, 326337. 
Umar, S., Asif, M., Sajad, M., Ansari, M., Hussain, U., Ahmad, W., Siddiqui, A., Ahmad, S. and Khan, H.A. 2012. Anti-inflammatory and antioxidant activity of Trachyspermum ammi seeds in collagen induced arthritis in rats. Int. J. Drug Dev. Res. 10, 210-219.

Upadhyay, S.N., Dhawan, S., Garg, S. and Talwar, G.P. 1992. Immunomodulatory effects of neem (Azadirachta indica) oil. Int. J. Immunopharmacol. 14, 1187-1193.

Usman, M.R.M., and Barhate, S. 2012. Phytochemical investigation and study of anti-inflammatory activity of Moringa oleifera Lam. Int. J. Pharm. Res. Dev. 3, 114-119.

Varshney, K.K., Varshney, M. and Nath, B. 2020. Molecular modeling of isolated phytochemicals from Ocimum sanctum towards exploring potential inhibitors of SARS coronavirus main protease and papain-like protease to treat COVID-19 3554371). Social Science Research Network; Available at SSRN: https://ssrn.com/abstract=3554371 (Accessed on 01 January 2021)

Vigila, A. and Baskaran, X. 2008. Immunomodulatory effect of coconut protein on cyclophosphamide induced immune suppressed swiss albino mice. Ethnobot. Leafl. 12, 1206-1212.

Vinothapooshan, G. and Sundar, K. 2011. Immunomodulatory activity of various extracts of Adhatoda vasica Linn. in experimental rats. Afr. J. Pharm. Pharmacol. 5, 306-310.

Vitali, L.A., Beghelli, D., Nya, P.C., Bistoni, O., Cappellacci, L., Damiano, S., Lupidi, G., Maggi, F., Orsomando, G., Papa, F. and Petrelli, D. 2016. Diverse biological effects of the essential oil from Iranian Trachyspermum ammi. Arab J. Chem. 9, 775786.

Wang, D., Feng, Y., Liu, J., Yan, J., Wang, M., Sasaki, J.I. and Lu, C. 2010. Black garlic (Allium sativum) extracts enhance the immune system. Medicinal and Aromatic Plant Sci. Biotech. 4, 37-40.

Weber, N.D., Andersen, D.O., North, J.A., Murray, B.K., Lawson, L.D. and Hughes, B.G. 1992. In-vitro virucidal effects of Allium sativum (garlic) extract and compounds. Planta Med. 58, 417-423.
Wiart, C., Kumar, K., Yusof, M., Hassan, H., Fauzi, Z. and Sulaiman, M. 2005. Antiviral properties of Entlabdene diterpenes of Andrographis paniculata Nees, inhibitors of herpes simplex virus type 1. Phytother. Res. 19, 1069-1070.

Wilson, D.K., Shyamala, G., Paulpandi, M., Narayanasamy, A., Siram, K., Karuppaiah, A. and Sankar, V. 2021. Development and characterization of phytoniosome nano vesicle loaded with aqueous leaf extracts of Justicia adhatoda and Psidium guajoava against dengue virus (DEN-2). J. Clust. Sci. 32, 297304.

Worldometer Coronavirus Update. 2021. Retrieved from https://www.worldometers.info/coronavirus/ (Accessed on April 28, 2021)

Xiong, Y., Rajoka, M.S.R., Mehwish, H.M., Zhang, M., Liang, N., Li, C. and He, Z. 2021. Virucidal activity of Moringa A from Moringa oleifera seeds against Influenza A Viruses by regulating TFEB. Int. Immunopharmacol. 95, 107561.

Younus, I., Siddiq, A., Ishaq, H., Anwer, L., Badar, S. and Ashraf, M. 2016. Evaluation of antiviral activity of plant extracts against foot and mouth disease virus invitro. Pak. J. Pharm. Sci. 29, 1263-1268.

Yue, G.G., Chan, B.C., Hon, P.-M., Lee, M.Y., Fung, K.P., Leung, P.-C. and Lau, C.B. 2010. Evaluation of invitro anti-proliferative and immunomodulatory activities of compounds isolated from Curcuma longa. Food Chem. Toxicol. 48, 2011-2020.

Zaher, K.S., Ahmed, W.M. and Zerizer, S.N. 2008. Observations on the biological effects of black cumin seed (Nigella sativa) and green tea (Camellia sinensis). Glob. Vet. 2, 198-204.

Zhou, C.Q. and Zhang, Y. 2008. Immunomodulatory effect of Indian ginseng root extract on immunodeficiency mice. China J. Chin. Mater. Medica 33, 2014-2018. 